\title{
Herkunft, Leistung und Bildungschancen im gegliederten Bildungssystem: Vertiefte PISA-Analyse unter Einbezug der kognitiven Grundfähigkeiten
}

\section{Erich Ramseier und Christian Brühwiler}

Seit dem Vorliegen der für die Schweiz ernüchternden PISA-Ergebnisse wird die Frage nach der Gerechtigkeit unserer Bildungssysteme wieder verstärkt diskutiert. Im vorliegenden Beitrag werden Aspekte der Chancengleichheit unter Berücksichtigung allgemeiner kognitiver Grundfähigkeiten detailliert untersucht. Neben dem Einfluss individueller Herkunftsmerkmale wird auch der Beitrag der Schule zur Erklärung fachlicher Leistungsunterschiede herangezogen. In die Analysen wurden die Daten von 2'054 Schülerinnen und Schülern der Kantone Bern und St. Gallen einbezogen, bei denen zusätzlich zu den PISA-Tests die kognitiven Grundfähigkeiten erfasst wurden. Die Ergebnisse zeigen, dass fachliche Leistungen auch unter Kontrolle der kognitiven Grundfähigkeiten stark von der sozialen Herkunft mitbestimmt werden. Eine entscheidende Rolle kommt dabei der Zugehörigkeit zu einem bestimmten Schultyp zu. Diese wird offensichtlich nicht nur durch fachliche Leistungen, sondern in hohem Ausmass über die soziale Herkunft der Schülerinnen und Schüler bestimmt. Überdies werden die schulischen Lernmöglichkeiten durch die soziokulturelle Zusammensetzung der einzelnen Schule beeinflusst. Diese Schuleffekte fallen unter Berücksichtigung der individuellen Voraussetzungen und des Schultyps jedoch deutlich geringer aus als die im internationalen PISA-Bericht $(O E C D, 2001)$ dargestellten Brutto-Effekte.

\section{Einleitung und Fragestellung}

Das mässige Abschneiden der Schweiz in der PISA-Studie (BFS/EDK, 2002) hat heftige Diskussionen über die Leistungsfähigkeit unseres Bildungswesens und um die Verantwortung für Bildungserfolge und Mängel entfacht. Neben dem bescheidenen mittleren Niveau der Leseleistung der schweizerischen 15-Jährigen gibt besonders zu denken, dass die Leistungsstreuung und vor allem der $\mathrm{Zu}$ sammenhang zwischen Leistung und sozialer sowie kultureller Herkunft in der Schweiz höher sind als in den meisten anderen Ländern (OECD, 2001). In der nationalen Auswertung zu PISA wurde der Einfluss der kulturellen (Moser, 
2002) sowie der sozialen Herkunft (Coradi-Vellacott \& Wolter, 2002) im Detail beschrieben und die hohe Bedeutung familiärer und kultureller Verhältnisse für den schulischen Erfolg nachgewiesen. Dabei blieben Unterschiede in den kognitiven Grundfähigkeiten unberücksichtigt. Ungeklärt ist ebenfalls, in welchem Masse die Schule zu diesem Ergebnis beiträgt.

Im Folgenden sollen die Abhängigkeit der Leistungen von kultureller und sozialer Herkunft sowie der Beitrag der Schule zur Divergenz der Leistungen genauer identifiziert werden. Dazu wird zusätzlich zu den PISA-Daten ein Mass für die kognitiven Grundfähigkeiten in die Analyse einbezogen, damit der Einfluss sozialer und kultureller Merkmale danach kontrolliert werden kann. Damit soll vermieden werden, dass die Schule für Unterschiede verantwortlich gemacht wird, die nicht oder nur marginal in ihrem Einflussbereich liegen.

In gegliederten Schulsystemen haben sich die Übergänge zwischen Bildungsstufen als kritisch für die Entstehung von sozialen Ungleichheiten erwiesen (Baumert \& Köller, 1998). Es soll deshalb besonders untersucht werden, wie sich Herkunftsmerkmale, kognitive Grundfähigkeiten und Schulleistungen im Selektionsprozess auswirken.

Die soziokulturelle Herkunft und die kognitiven Grundfähigkeiten der Schülerinnen und Schüler üben nicht nur als Individualmerkmale einen Einfluss auf das Lernen und die Leistungen aus. Unterscheiden sich Schulen in der Zusammensetzung ihrer Schülerschaft, so ergeben sich daraus auch unterschiedliche Lernmilieus und kollektive Effekte auf die Leistungen. Ein weiteres Ziel dieses Artikels ist deshalb, die Leistungsunterschiede zwischen einzelnen Schulen unter Berücksichtigung der Schultypen und der kognitiven Grundfähigkeiten der Schülerinnen und Schüler zu differenzieren, um so Selektionseffekte und direkte Wirkungen der Schulen besser aufzuschlüsseln zu können.

Diese Thematik wird im Folgenden näher begründet und in drei Fragestellungen gegliedert. Nach der Präsentation des Vorgehens werden die Ergebnisse entsprechend diesen drei Fragestellungen dargestellt und diskutiert.

\section{Schulische Leistung als Folge von Herkunft und kognitiven Grundfähigkeiten}

Helmke und Weinert (1997) sprechen von der multiplen Determiniertheit schulischer Leistungen. Neben schulischen und familiären Faktoren spielen insbesondere soziokulturelle Rahmenbedingungen und individuelle Voraussetzungen eine wichtige Rolle. Dabei setzt die soziale und kulturelle Herkunft den Rahmen, in dem Lernprozesse stattfinden.

Zahlreiche empirische Untersuchungen haben die hohe Bedeutung kognitiver Grundfähigkeiten bzw. der Intelligenz für schulische Leistungen gezeigt (Helmke \& Weinert, 1997). So konnten Artelt, Stanat, Schneider und Schiefele (2001) anhand der deutschen PISA-Daten aufzeigen, dass die kognitiven Grundfähigkeiten - ohne Berücksichtigung des bereichsspezifischen Vorwissens - eindeutig der beste Prädiktor für die Lesekompetenz der Schülerinnen und 
Schüler sind. Allerdings besteht in Bezug auf eine allgemein akzeptierte Definition von Intelligenz noch wenig Einigkeit. Auch neuere Intelligenzdefinitionen gehen nach Weinert (1997) in der Regel auf die vage und doch gehaltvolle Definition von Binet und Simon (1905) zurück. Demnach seien "gut urteilen, gut verstehen, gut denken» (Weinert, 1997, 137) die wesentlichen Funktionen der Intelligenz. Obschon sowohl Begriff als auch Erfassung der Intelligenz umstritten sind, kann weder in der psychologischen Diagnostik noch in der empirisch ausgerichteten Lehr-Lernforschung auf die Erfassung der Intelligenz mittels IQTests verzichtet werden.

Heftige, häufig ideologisch überlagerte Kontroversen hat auch die AnlageUmwelt-Debatte ausgelöst (Herrnstein \& Murray, 1994; Klauer, 1998; Rhyn, 1995). Heutzutage wird anerkannt, dass Intelligenz nicht nur durch Umweltfaktoren beeinflusst wird, sondern zu einem erheblichen Anteil genetisch bedingt ist. Diese Erkenntnis geht hauptsächlich auf Zwillings- und Adoptionsstudien zurück, die erhebliche Erbeinflüsse auf allgemeine intellektuelle Fähigkeiten nachweisen konnten (z.B. Plomin, 1988). Nach Plomin gibt es keine andere Variable, die soviel Varianz der Intelligenz aufklärt wie die Vererbung. Die additive Zerlegung von Varianzanteilen wird allerdings der Interaktion von Anlage und Umwelt nicht gerecht (Zimmermann \& Spangler, 200); differenzierte Modelle zeigen, dass erhebliche Umwelt-einflüsse auch mit sehr hoher Erblichkeit verträglich sind (Dickens \& Flynn, 2001). Umstritten ist, ob kristalline bzw. fluide Intelligenz (Cattell, 1971) unterschiedlich durch Vererbung determiniert ist und inwieweit trotz kultureller Einflüsse faire Tests möglich sind (Guthke, 1998).

Im Zusammenhang mit den Einflussmöglichkeiten der Schule ist vor allem die Frage nach der Veränderbarkeit kognitiver Grundfähigkeiten von Interesse. Weinert (1997) betont die hohe Stabilität intellektueller Fähigkeiten zwischen Individuen von der frühen Kindheit an. Intelligenz im Sinne allgemeiner kognitiver Fähigkeiten lässt sich demnach kaum durch spezifische Lernprogramme verbessern. Nach Guthke (1998, S. 7) ist jedenfalls kaum daran zu zweifeln, dass die Qualität des Unterrichts das schulische Wissen mehr beeinflusst als die Intelligenzleistungen.

Vor diesem Hintergrund soll als erste Fragestellung geklärt werden, wie und in welchem Ausmass personale und soziokulturelle Merkmale mit der schulischen Leistung zusammenhängen. Als Einflussgrössen werden die kognitiven Grundfähigkeiten, die soziale und kulturelle Herkunft sowie das Geschlecht herangezogen. Von Interesse ist dabei vor allem, wie weit Herkunftsvariablen nach Kontrolle der kognitiven Grundfähigkeiten Leistung voraussagen. Zusätzlich wird der Schultyp in das Erklärungsmodell aufgenommen, um direkte und über den Schultyp vermittelte Effekte der Herkunftsvariablen zu identifizieren. Um zu klären, ob die Effekte fachspezifisch variieren, werden die Modelle sowohl in Bezug auf die Leseleistung als auch für mathematische und naturwissenschaftliche Leistungen berechnet. Da Lesen in der Konzeption von PISA als grundlegende Kompetenz betrachtet wird, die insbesondere für den selbständigen Wissenser- 
werb auf der Basis von Texten wesentlich ist (OECD, 2001), kann Lesen als eine fächerübergreifende Kompetenz aufgefasst werden. In diesem Sinne wird Lesen als Prädiktor für mathematische und naturwissenschaftliche Leistungen eingeführt. Damit kann der Beitrag der Lesefähigkeit für fachbezogene Leistungen unter Kontrolle der kognitiven Grundfähigkeiten und der soziokulturellen Herkunft geschätzt werden.

\section{Chancengleichheit bei Selektionsprozessen}

Schullaufbahnen, Selektionsprozesse und damit auch Chancengleichheit hängen von der Gliederung eines Bildungssystems in unterschiedliche Bildungsgänge ab. International überwiegen auf der Sekundarstufe I einheitliche, integrierte Systeme. In der Schweiz sind dagegen gegliederte Schulsysteme häufiger, die in Bildungsgänge (Schultypen) mit unterschiedlichen Anspruchsniveaus und Zielen unterteilt sind. Je nach kantonalen und lokalen Varianten werden dabei die Schultypen innerhalb einer Schule oder in getrennten Schulen angeboten und die Kooperation dazwischen kann unterschiedlich eng sein (vgl. Bain, Favre, Hexel, Lurin \& Rastoldo, 2000; zum aktuellen Stand in der Schweiz siehe www.edk.ch).

Besonders in den gegliederten Schulsystemen ist die Unterscheidung zwischen primären und sekundären sozialen Ungleichheiten wichtig, wie sie Baumert und Schümer (2001) in Anlehnung an Breen und Goldthorpe (1997) sowie Boudon (1974) beschreiben. Als primäre Ungleichheiten werden von der sozialen Herkunft abhängige Unterschiede in den bisher erworbenen und für die nächste Bildungsstufe notwendigen Kompetenzen bezeichnet. Unter sekundären Ungleichheiten werden soziale Disparitäten der Bildungsbeteiligung, konkret des Besuchs weiterführender Schulen, verstanden, die trotz gleicher Kompetenzen auftreten. Zutavern, Brühwiler und Biedermann (2002) wiesen nach, dass selbst bei gleichen fachlichen Leistungen der Besuch eines höheren Schultyps stark schichtabhängig ist. In Deutschland stellten Baumert und Schümer (2002) vor allem beim Gymnasialbesuch solche sekundären sozialen Ungleichheiten fest. Diese unterschiedliche Nutzung von Bildungsangeboten führt zu einer Reproduktion von herkunftsbedingten Ungleichheiten im Bildungssystem (Baumert \& Schümer, 2001). Das Ausmass dieser sekundären sozialen Ungleichheiten gibt Auskunft über die Chancengleichheit an kritischen Übergängen in einem Bildungssystem.

Wer indessen im gegliederten Schulsystem einen bestimmten Bildungsgang gewählt und die Eingangsbedingungen erfüllt hat, wird kaum mehr auf Grund der sozialen Herkunft benachteiligt (Baumert, Köller \& Schnabel, 2000; Helmke, Hosenfeld, Schrader \& Wagner, 2002). Zahlreiche Studien zeigen jedoch, dass sich typenspezifische Milieus entwickeln. Diese führen in einem Scheren-Effekt dazu, dass die Leistung in den Typen mit höheren Anforderungen auch nach Kontrolle von Eingangsleistung, sozialer Herkunft und kognitiver Grundfähigkeiten rascher zunimmt als in den Typen mit Basisansprüchen (Bau- 
mert et al., 2000, Gamoran \& Berends, 1987; Gamoran, 1992; Moser \& Rhyn, 1999, 100). Die Wirkung der schulformspezifischen Milieus wandelt damit beim Übertritt vorhandene sekundäre Ungleichheiten in Kompetenzunterschiede und damit in primäre Chancenungleichheiten um. Echte Chancengleichheit würde dagegen bedeuten, dass über alle Stufen der Ausbildung hinweg allein die erbrachten individuellen Leistungen über die Bildungsbeteiligung, Bildungsabschlüsse und schliesslich Berufs- und Lebenschancen entscheiden.

Als zweite Fragestellung interessiert somit die Rolle der individuellen Einflussfaktoren bei der Wahl bzw. der Zuteilung zu einem Schultyp. Dabei interessiert die Leseleistung nicht mehr als Indikator von Lernerfolg sondern wird als Voraussetzung für das Absolvieren eines bestimmten Schultyps angesehen. Damit lässt sich prüfen, ob die soziale und kulturelle Herkunft bei gleichen intellektuellen Voraussetzungen die Bildungschancen beeinflusst oder ob Bildungschancen unabhängig davon verteilt sind. Gefragt wird auch, ob sich Chancenungleichheiten beim Übertritt in die Sekundarschule bzw. ins Gymnasium voneinander unterscheiden.

\section{Einfluss der einzelnen Schulen auf fachliche Leistungen}

Das Leistungsniveau, die Leistungsstreuung und der Zusammenhang zwischen Herkunft und Leistung in der Schülerpopulation beschreiben die Qualität des Bildungswesens bezüglich der Kriterien Effektivität und Gleichheit. Wenn es allerdings darum geht, die Prozesse zu verstehen, die zu diesen Effekten des Bildungssystems führen, so genügt die Analyse auf der Ebene von Individuen nicht. Die Schulwirksamkeitsforschung (school effectiveness research) hat gezeigt, dass die einzelne Schule als Organisationseinheit eine wesentliche Rolle für die Vermittlung von Bildung spielt (z.B. Scheerens \& Bosker, 1997). Neben der Schule ist aber auch die Klasse, in der sich der konkrete Unterrichtsprozess abspielt, zu beachten. Unterrichtsbedingungen bzw. Unterschiede zwischen Klassen haben sich oft als gewichtiger erwiesen als Bedingungen der Schulorganisation bzw. Unterschiede zwischen Schulen (Ditton \& Krecker, 1995; Scheerens \& Bosker, 1997, 303f). Das Bildungssystem funktioniert als eine hierarchische Struktur mit den Ebenen Gesamtsystem (z.B. Definition des Curriculums und der Schulstruktur), Schule, Klasse und Individuum und sollte entsprechend unter Berükksichtigung dieser vier Ebenen analysiert werden.

Das Design von PISA erlaubt, Unterschiede zwischen den Schulen zu untersuchen. ${ }^{1}$ Im ersten internationalen Bericht wird denn auch festgestellt, dass sich Länder stark darin unterscheiden, ob die Leistungsunterschiede zwischen Schulen gross sind im Vergleich zu Leistungsunterschieden zwischen Schülerinnen und Schülern innerhalb einer Schule (OECD, 2001, S. 69).

Schulen unterscheiden sich unter anderem in der sozialen und kulturellen Zusammensetzung ihrer Schülerschaft. Diese soziale Segregation kann auf die unterschiedliche Zusammensetzung der Bevölkerung im Einzugsgebiet der 
Schule oder auf Praktiken der Selektion und der Schulwahl zurückzuführen sein. Die PISA-Ergebnisse zeigen, dass die soziale Zusammensetzung der Schule oft einen stärkeren Effekt auf die Leistung hat als die individuelle soziale Herkunft eines Schülers bzw. einer Schülerin (OECD, 2001, S. 237). Auch für die kulturelle Zusammensetzung wurden Effekte auf das Leistungsniveau festgestellt (Moser \& Rhyn, 2000, S. 100). In der Tendenz weisen Länder, die Schülerinnen und Schüler schon früh bestimmten Schultypen zuweisen, grosse Schulunterschiede und einen hohen Zusammenhang zwischen Herkunft und Leistung auf (OECD, 2001, S. 77).

Die im internationalen Bericht dargestellten Ergebnisse zu den Schuleffekten und zum Einfluss der sozialen Zusammensetzung von Schulen sagen für die Schweiz jedoch wenig aus. Die schweizerische PISA-Population der 15-Jährigen verteilt sich je nach Sprachregion in unterschiedlichem Verhältnis auf Schulen der Sekundarstufe I (Realschulen, Sekundarschulen usw.) und der Sekundarstufe II (Berufsschulen, verschiedene allgemein bildende Schulen). Schulmerkmale wie etwa die Schulgrösse haben somit keine einheitliche Bedeutung, und der Anteil der Varianz zwischen Schulen hängt in nicht interpretierbarer Weise vom Mischungsverhältnis zwischen Schulen der Sekundarstufe I bzw. II und innerhalb der Sekundarstufe I von der Mischung zwischen Schulen ab, die nur einen Typ anbieten, die mehrere Typen anbieten oder auf die Typeneinteilung verzichten. Um für die Schweiz Aussagen machen zu können, drängt sich deshalb eine Analyse der Schuleffekte auf, die sich auf die 9. Klassen bezieht und die Struktur der Schultypen sowie die kognitiven Grundfähigkeiten explizit berücksichtigt.

Die dritte Fragestellung dreht sich somit um die Analyse des Einflusses der einzelnen Schulen auf die Leseleistung. Dabei geht es erstens darum, die Schuleffekte unter Berücksichtigung des Schultyps und individueller Herkunftsmerkmale genauer zu beschreiben (Unterscheidung von Brutto- und Netto-Effekten). Dabei interessiert, ob auch nach Kontrolle von Schultyp und wichtigen Individualmerkmalen Effekte nachgewiesen werden können, die als Wirkung der einzelnen Schulen anzusehen sind.

Zweitens soll untersucht werden, ob ein erheblicher Effekt des sozialen und kulturellen Milieus der Schule auf die Leistungsergebnisse festzustellen ist, wenn der Schultyp und Individualmerkmale kontrolliert werden, und ob bestimmte Merkmale der Schul- und Lernsituation identifiziert werden können, die einen Effekt auf das Leistungsniveau der Schule haben.

\section{Methode und deskriptive Ergebnisse}

\section{Mess instrumente}

\section{Messung der Grundbildung in PISA}

PISA misst die Grundfähigkeiten von 15-Jährigen in den Fachbereichen Lesen, Mathematik und Naturwissenschaften (OECD, 2001). In der Schweiz wurden 
zusätzlich die Schülerinnen und Schüler aus den 9. Klassen in die Erhebung einbezogen, um so das Wissen und die Fähigkeiten der Schweizer Jugendlichen am Ende der obligatorischen Schulzeit zu erfassen. Dabei geht es nicht um das Abfragen spezifischen Wissens oder Schulstoffs, sondern um die Beherrschung von Prozessen und das Verstehen von Konzepten in realitätsnahen Situationen (Lite$\operatorname{racy}^{2}$ ). Diese «Literacy» oder Grundbildung steht somit zwischen fachbezogenen und fächerübergreifenden Konzepten. Es wurden Kompetenzen erfasst, die als grundlegend für das erfolgreiche Bewältigen künftiger Aufgaben im Erwachsenenleben erachtet werden. Die gemessenen Kompetenzen wurden so auf einer Skala normiert, dass der Mittelwert der 15-Jährigen aller OECD-Länder 500 Punkte beträgt und die Leistungen von zwei Dritteln der Jugendlichen im Bereich zwischen 400 und 600 Punkten liegen $(S D=100) .3$

Lesekompetenz wird im Rahmen von PISA als Fähigkeit verstanden, geschriebene Texte zu verstehen, deren zentrale Aussagen zu identifizieren sowie den Inhalt oder die Form eines Textes zu interpretieren und kritisch zu bewerten. Die Testaufgaben waren so konstruiert, dass sie in Bezug auf Textformen und Lesesituationen eine Vielzahl von verschiedenen Leseanlässen abdecken. Die Aufgaben umfassten sowohl zusammenhängende Prosatexte (z.B. Erzählungen oder Argumentationen) als auch «nicht-kontinuierliche» Texte wie Tabellen, Grafiken oder Diagramme. Die Aufgabentexte variierten zudem in Bezug auf den Verwendungskontext. Es mussten beispielsweise Texte zu persönlichen, beruflichen oder öffentlichen Anlässen bearbeitet werden.

Die mathematische Grundbildung umfasst die Fähigkeit, mathematische Probleme zu identifizieren, zu verstehen sowie fundierte mathematische Urteile abzugeben. Geprüft wurde das Beherrschen mathematischer Schlüsselkonzepte bis hin zum komplexen mathematischen Verständnis. Die naturwissenschaftliche Grundbildung wird als die Fähigkeit verstanden, naturwissenschaftliches Wissen anzuwenden, naturwissenschaftliche Fragen zu erkennen und daraus Schlussfolgerungen zu ziehen. Es wurde geprüft, inwieweit naturwissenschaftliche Konzepte auf wissenschaftliche Fragen aus dem Alltag, etwa zu aktuellen Themen wie Erde, Umwelt oder Technologie, angewendet werden können.

\section{Kognitive Grundfäbigkeiten}

Die allgemeinen kognitiven Grundfähigkeiten wurden mit je einer verbalen und figuralen Subskala des Kognitiven Fähigkeits-Tests (KFT) von Heller, Gaedike und Weinläder (1985) erfasst. Die Testaufgaben prüfen auf der Basis von Begriffen bzw. Figuren die Fähigkeit, inwieweit korrekte Analogieschlüsse gezogen werden können. Im Unterschied zu fachspezifischem Wissen sind solche stärker genetisch bestimmte allgemeine intellektuelle Fähigkeiten weniger stark durch schulisches Lernen beeinflussbar (Guthke, 1998). In Übereinstimmung mit Heller et al. (1985) kann davon ausgegangen werden, dass der Zuwachs an figuralen kognitiven Grundfähigkeiten weniger von soziokulturellen Unterschieden beeinflusst wird und damit durch sie eher frühe Begabungspotentiale abgebildet 
werden als durch verbale Grundfähigkeiten. Deren Entwicklung geht stärker mit einem mehr oder weniger reichhaltigen sprachlichen Anregungsgehalt der Umwelt einher. Verbale kognitive Grundfähigkeiten dürften somit mit zunehmendem Alter vermehrt Anteile einschliessen, die durch die soziale Herkunft bedingt sind. Dass die verbale Subskala, die ein differenziertes Verständnis deutscher Begriffe voraussetzt, stärker mit der Herkunft konfundiert ist, wird besonders im Falle der kulturellen Herkunft (Fremdsprachigkeit) offensichtlich. Aus diesem Grund wurde für die nachfolgenden Analysen dort, wo es um die Abgrenzung zwischen Herkunft und kognitiven Grundfähigkeiten geht, ausschliesslich der figurale KFT-Subtest zur Abbildung der allgemeinen kognitiven Grundfähigkeiten verwendet.

\section{Soziale Herkunft}

Die soziale Herkunft von Jugendlichen wird im Wesentlichen dadurch bestimmt, welche ökonomischen, kulturellen und sozialen Ressourcen ihnen zur Verfügung stehen (Bourdieu, 1983). Eine der Stärken von PISA ist die detaillierte Erfassung dieser sozialen Verhältnisse, über die die Jugendlichen in einem umfangreichen Begleitfragebogen Auskunft geben mussten. Als Mass für die soziale Herkunft wurde ein Index gebildet, der die Indikatoren Beruf und Ausbildung der Eltern sowie die Bildungsnähe des Elternhauses, erfasst durch die Anzahl Bücher und durch das Vorhandensein anderer kultureller Ressourcen (z.B. klassische Literatur), einschliesst.

\section{Kulturelle Herkunft}

Die kulturelle Herkunft wird über ein Merkmal erfasst, das sich aus dem Sprachhintergrund und dem Geburtsort zusammensetzt. Auf Grund der Sprache, welche die Jugendlichen normalerweise zu Hause sprechen, werden die beiden Gruppen Deutschsprachige und Fremdsprachige gebildet, wobei letztere wiederum nach dem Geburtsort - in der Schweiz oder im Ausland geboren - unterschieden werden. ${ }^{4}$ Die in der Schweiz geborenen Jugendlichen mit nicht-deutschsprachigem Sprachhintergrund können in ihrer Mehrheit vereinfacht als Jugendliche der zweiten Generation bezeichnet werden. Im Ausland geborene Fremdsprachige sind dagegen erst im Verlaufe ihrer Kindheit immigriert und haben oft nur einen Teil ihrer Schulzeit in der Schweiz verbracht. Schülerinnen und Schüler, die sich weniger als ein Jahr im deutschen Sprachraum aufhalten, sind von der Erhebung ausgeschlossen worden.

\section{Schultyp}

Die Schulstrukturen der beiden untersuchten Kantone Bern und St. Gallen unterscheiden sich auf der Sekundarstufe I nur unwesentlich voneinander (vgl. Zutavern et al., 2002). Beide Kantone selektieren die Schülerinnen und Schüler nach sechs Primarschuljahren in eine dreigliedrige Oberstufe. Die drei Schultypen unterscheiden sich in ihrem Anforderungsniveau: Realschule (Grundanfor- 
derungen), Sekundarschule (erweiterte Anforderungen) und Gymnasium 5 bzw. Kantonsschule (hohe Anforderungen). Vielerorts befinden sich die Klassen der Realschule und der Sekundarschule in demselben Schulgebäude und bilden ein gemeinsames Oberstufenzentrum. Die Zusammenarbeit zwischen den beiden Schultypen ist aber dennoch meist marginal. Im Kanton Bern werden allerdings auch Schulmodelle mit einer ausgeprägten Kooperation zwischen den Schultypen geführt. Die beiden Kantone Bern und St. Gallen repräsentieren demnach jene Schulform, die in der Schweiz nach wie vor typisch ist. Weitere Schultypen wie Klein- und Sonderklassen sowie nicht staatlich unterstützte Privatschulen wurden nicht in die Untersuchung einbezogen.

\section{Beschreibung der Stichprobe und Durchführung der Erhebung}

Der Test zur Erfassung der kognitiven Grundfähigkeiten mittels KFT ist eine Erweiterung von PISA 2000. In der Schweiz haben einzig die beiden Kantone Bern und St. Gallen diesen Zusatztest durchgeführt. Die Durchführung des KFT erfolgte jeweils am Ende der ordentlichen PISA-Testsitzung, die gemäss international festgelegter Richtlinien stattfand (vgl. OECD, 2001). Da die Schülerinnen und Schüler beim Absolvieren des KFT bereits mehr als zwei Stunden Testzeit hinter sich hatten, dürfte mit den Testergebnissen das tatsächliche kognitive Potential eher etwas unterschätzt werden. Sämtliche Schülerinnen und Schüler absolvierten den KFT jedoch unter denselben Umständen, weshalb die suboptimalen Testbedingungen für die hier beabsichtigte Erklärung von Leistungsunterschieden kaum ins Gewicht fallen dürften.

Tabelle 1: Verteilung der Stichprobe nach Geschlecht, Schultyp und kultureller Herkunft (ungewichtet)

\begin{tabular}{|l|c|c|c|c|c|c|c|}
\hline & \multicolumn{3}{|c|}{ Schultyp } & \multicolumn{2}{c|}{ Kulturelle Herkunft } & N \\
\hline & $\begin{array}{c}\text { Real- } \\
\text { schule }\end{array}$ & $\begin{array}{c}\text { Sekundar- } \\
\text { schule }\end{array}$ & $\begin{array}{c}\text { Gymna- } \\
\text { sium }\end{array}$ & $\begin{array}{c}\text { deutsch- } \\
\text { sprachig }\end{array}$ & \multicolumn{2}{c|}{ fremdsprachig } & \\
\hline & & & & & $\begin{array}{c}\text { in der } \\
\text { Schweiz } \\
\text { geboren }\end{array}$ & $\begin{array}{c}\text { im } \\
\text { Ausland } \\
\text { geboren }\end{array}$ & \\
\hline Mädchen & $26 \%$ & $49 \%$ & $25 \%$ & $86 \%$ & $7 \%$ & $7 \%$ & $1^{\prime} 044$ \\
\hline Knaben & $34 \%$ & $43 \%$ & $23 \%$ & $87 \%$ & $7 \%$ & $6 \%$ & $1^{\prime} 010$ \\
\hline Total & $30 \%$ & $46 \%$ & $24 \%$ & $86 \%$ & $7 \%$ & $6 \%$ & $2^{\prime} 054$ \\
\hline
\end{tabular}


Die Stichprobe umfasst insgesamt 2'054 Neuntklässlerinnen und Neuntklässler aus total 73 Schulen der Kantone Bern und St. Gallen. ${ }^{6}$ Tabelle 1 zeigt die Verteilung der in die Analysen einbezogenen Schülerinnen und Schüler nach Geschlecht, Schultyp und kultureller Herkunft. Bei der kulturellen Herkunft ist zu beachten, dass die drei Gruppen unterschiedlich stark besetzt sind. Die Anteile bei der Schultypenzugehörigkeit sind ausgewogener, wobei fast die Hälfte der Schülerinnen und Schüler eine Sekundarschule und etwa ein Viertel ein Gymnasium besuchen. Nennenswerte Geschlechterunterschiede zeigen sich einzig beim höheren Mädchenanteil in den Sekundarschulen bzw. dem höheren Knabenanteil in den Realschulen.

Da das Ziel der vorliegenden Analyse nicht darin besteht, deskriptive Aussagen über die ganze Population der beiden Kantone zu machen, sondern Zusammenhänge zu analysieren, werden die Daten ungewichtet verwendet, um so ihren Informationsgehalt optimal auszunützen. ${ }^{7}$

\section{Deskriptive Ergebnisse}

Auf Grund der Reliabilitätsanalyse wurde ein Item des für diese Analyse hauptsächlich verwendeten figuralen Fähigkeitstests ausgeschlossen. Mit den verbleibenden 24 Items ergibt sich ein Cronbach-alpha-Wert von .83. Die Testleistungen sind allerdings nicht normalverteilt. Durchschnittlich wurden 71 Prozent der Aufgaben richtig gelöst $(M=71.2 ; S D=19.3), 25$ Prozent der Jugendlichen haben mehr als 85 Prozent der Aufgaben richtig beantwortet. 34 Schülerinnen und Schüler konnten sämtliche Aufgaben korrekt lösen. Dies deutet darauf hin, dass der Test eher zu leicht war (Ceiling-Effekt). Die Schülerinnen und Schüler im oberen Intelligenzbereich konnten möglicherweise nicht ihr ganzes Fähigkeitspotential ausschöpfen. Beim verbalen Fähigkeitstest wurden zwei Items ausgeschlossen. Der Test aus den verbleibenden 18 Items weist Werte von $\alpha=.74$; $\mathrm{M}=53.0$ und $\mathrm{SD}=20.2$ auf.

Um die Effekte in den nachfolgenden Analysen besser einordnen zu können, werden hier die Zusammenhänge zwischen den kognitiven Grundfähigkeiten sowie den im PISA-Test erzielten fachlichen Leistungen und den Schultypen sowie der kulturellen Herkunft dargestellt (vgl. Tabelle 2). Deutlich sind die Mittelwertsunterschiede zwischen den einzelnen Schultypen. Dieses Ergebnis vermag auf Grund des selektiven Schulsystems in den beiden Kantonen nicht weiter zu erstaunen. Die Differenz zwischen Realschule und Sekundarschule ist sowohl beim KFT als auch in den fachlichen Leistungen deutlich ausgeprägter als zwischen Sekundarschule und Gymnasium. Dieser Befund konnte auch für die gesamte Deutschschweiz bestätigt werden, wobei es trotz der grossen Mittelwertsunterschiede zu erheblichen Leistungsüberlappungen zwischen den Schultypen kommt (vgl. Zutavern et al., 2002). 
Tabelle 2: Kognitive Grundfähigkeiten und fachliche Leistungen nach Schultyp und kultureller Herkunft

\begin{tabular}{|l|c|c|c|c|c|c|}
\hline \multicolumn{1}{|c|}{ Schultyp } & \multicolumn{2}{c|}{ Realschule } & \multicolumn{2}{c|}{ Sekundarschule } & \multicolumn{2}{c|}{ Gymnasium } \\
\hline & M & SD & M & SD & M & SD \\
\hline KFT (figural) & 56.9 & 21.1 & 75.2 & 15.1 & 81.1 & 13.3 \\
\hline Leseleistung & 426 & 70 & 533 & 63 & 590 & 67 \\
\hline Mathematikleistung & 463 & 72 & 564 & 66 & 611 & 68 \\
\hline $\begin{array}{l}\text { Naturwissenschaft- } \\
\text { liche Leistung }\end{array}$ & 433 & 68 & 528 & 68 & 592 & 67 \\
\hline
\end{tabular}

\begin{tabular}{|l|c|c|c|c|c|c|}
\hline Kulturelle Herkunft & \multicolumn{3}{|c|}{ deutschsprachig } & \multicolumn{2}{c|}{$\begin{array}{c}\text { fremdsprachig, in der } \\
\text { Schweiz geboren }\end{array}$} & $\begin{array}{c}\text { fremdsprachig, im } \\
\text { Ausland geboren }\end{array}$ \\
\hline & M & SD & M & SD & M & SD \\
\hline KFT (figural) & 72.5 & 18.6 & 66.9 & 19.1 & 57.6 & 22.7 \\
\hline Leseleistung & 526 & 84 & 473 & 88 & 417 & 98 \\
\hline Mathematikleistung & 558 & 81 & 491 & 92 & 445 & 96 \\
\hline $\begin{array}{l}\text { Naturwissenschaft- } \\
\text { liche Leistung }\end{array}$ & 525 & 84 & 478 & 92 & 414 & 89 \\
\hline
\end{tabular}

Die Abstände der fachlichen Leistungen in den drei Gruppen der kulturellen Herkunft fallen ebenfalls deutlich aus. Da es sich für die fremdsprachigen Schülerinnen und Schüler eigentlich um einen Test in einer Zweitsprache handelte, sind die Unterschiede zu erwarten. Weil die Leistungsdifferenzen zwischen den einzelnen Kategorien ungefähr gleich gross sind, ist es vertretbar, die kulturelle Herkunft in den nachfolgenden Analysen als lineare Variable zu behandeln. Die deutlich höhere Standardabweichung bei den fremdsprachigen Jugendlichen weist zudem darauf hin, dass es sich hierbei keineswegs um eine homogene Gruppe handelt (vgl. Moser, Ramseier \& Berweger, 2002).

In den bisherigen PISA-Analysen ist die enge Beziehung der kulturellen und insbesondere der sozialen Schichtzugehörigkeit zur Leistung immer wieder betont worden, der Zusammenhang zu den kognitiven Grundfähigkeiten konnte dabei jedoch nicht mit berücksichtigt werden. Obwohl es sich bei der figuralen KFT-Skala um einen nicht-sprachlichen Test handelt, zeigen sich signifikante Korrelationen mit der sozialen $(r=.29)$ und der kulturellen $(r=-.19)$ Herkunft. Diese Konfundierung wird in den folgenden Analysen mit berücksichtigt und damit die soziokulturellen Effekte vom Einfluss allgemeiner intellektueller Fähigkeiten bereinigt. 


\section{Erklärungsmodelle zur Vorhersage von schulischer Leistung}

Der Erwerb von fachlichen Kompetenzen hängt stets von einem ganzen Bündel von Faktoren ab, die grob eingeteilt der Schule, dem lernenden Individuum oder dem soziokulturellen Umfeld des Lernenden zugeordnet werden können (Helmke \& Weinert, 1997). Die bisherigen PISA-Befunde zeigen einen starken Zusammenhang der sozialen und kulturellen Herkunft mit fachbezogenen Leistungen, der im Folgenden anhand von Pfadmodellen unter der Berücksichtigung kognitiver Grundfähigkeiten analysiert wird. Als weiteres individuelles Merkmal wird das Geschlecht mit in das Erklärungsmodell aufgenommen. In diesen Pfadmodellen werden die Korrelationen zwischen den Variablen aufgrund theoretischer Annahmen als Ausdruck kausaler Beeinflussung interpretiert und die zu Grunde liegenden direkten Effekte (standardisierte Regressionskoeffizienten) identifiziert. ${ }^{8}$ Abbildung 1 bildet die statistisch signifikanten Zusammenhänge des Modells für die Leseleistung ab. Der Messfehler für die beiden Variablen kognitive Grundfähigkeiten und soziale Herkunft wurde im Modell berücksichtigt, indem die empirisch festgestellte Reliabilität gesetzt wurde (Jöreskog \& Sörbom, 1988, S. 153).

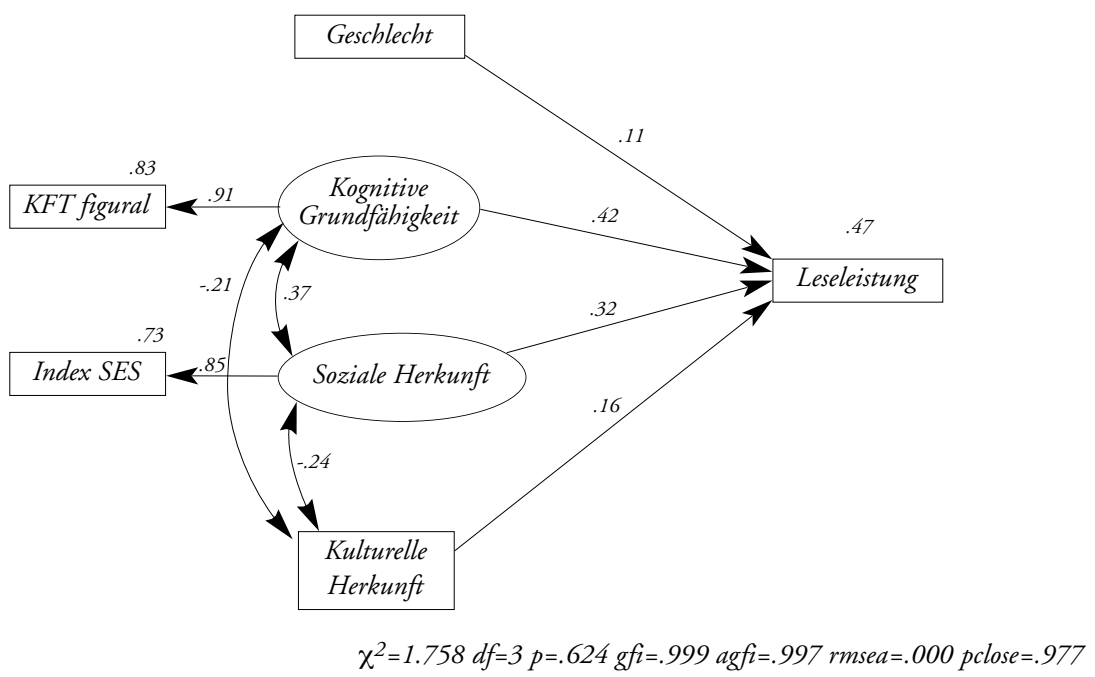

Abbildung 1: Erklärung der Leseleistung unter Einbezug kognitiver Grundfähigkeiten $(N=1026) 9$

Anmerkung: Das Geschlecht wurde als $0=$ Knaben und $1=$ Mädchen kodiert, die kulturelle Herkunft als 1 = deutschsprachig, 2 = fremdsprachig, in der Schweiz geboren und 3 = fremdsprachig, im Ausland geboren. SES = Index der sozialen Herkunft 
Insgesamt erklären die vier Faktoren 47 Prozent der Varianz in der Leseleistung. Alle Bedingungsfaktoren erweisen sich als signifikant, wobei die kognitive Grundfähigkeit - abzulesen am grössten angegebenen Pfadkoeffizienten $(\beta=.42)$ - den engsten Zusammenhang mit der Leseleistung aufweist. Die soziale Herkunft der Schülerinnen und Schüler hat ebenfalls einen beträchtlichen eigenständigen Einfluss auf die Leistung im Lesen. Weniger eng, aber noch immer bedeutsam, sind die Beziehungen zwischen der kulturellen Herkunft bzw. dem Geschlecht und der Lesefähigkeit. Der positive Koeffizient beim Geschlecht bedeutet, dass die Mädchen über höhere Lesekompetenzen verfügen als die Knaben. Die fehlenden Pfeile zwischen dem Geschlecht und den übrigen Einflussgrössen geben an, dass weder bezüglich kognitiver Grundfähigkeiten noch sozialer und kultureller Herkunft Geschlechterunterschiede bestehen. Die übrigen Prädiktoren beeinflussen sich jeweils gegenseitig, wobei die kognitive Grundfähigkeit - gemessen mit dem figuralen Subtest des KFT - deutlich stärker mit der sozialen als mit der kulturellen Herkunft zusammenhängt.

Ersetzt man in einem Alternativmodell den figuralen Fähigkeitstest durch die verbale Skala des KFT, so zeigt sich ein deutlich höherer Zusammenhang zwischen der verbalen Grundfähigkeit und der sozialen Herkunft (.53). Dies bestätigt die Annahme, dass verbale Grundfähigkeiten stärker durch das soziokulturelle Umfeld geprägt werden und entsprechend enger mit der Sozialschichtzugehörigkeit verbunden sind als figurale Fähigkeiten.

Das erste Modell zeigt die um die gegenseitige Konfundierung bereinigten Effekte der Herkunftsmerkmale auf die Leseleistung. Da die kognitiven Grundfähigkeiten und das soziokulturelle Umfeld die Lernleistung schon im frühkindlichen Alter und lange vor der Selektion in einen bestimmten Schultyp beeinflussen (Weinert, 1997) und der besuchte Schultyp eine Schlüsselrolle für die weitere Schullaufbahn spielt, wird im zweiten Modell der Schultyp als vermittelnde Variable zwischen den personalen und soziokulturellen Merkmalen und der Leseleistung mit aufgenommen (vgl. Abbildung 2).

Mit der Aufnahme des Schultyps kann die erklärte Gesamtvarianz auf 57 Prozent erhöht werden. Auffallend ist bei Berücksichtigung des Schultyps die deutliche Abnahme des direkten Schichteffekts auf die Leseleistung $(\beta=.14)$, der nun hauptsächlich indirekt über den Schultyp $(\beta=.44)$ verläuft. Der Besuch eines bestimmten Schultyps hängt selbst bei gleichen kognitiven Grundfähigkeiten stark von der jeweiligen Schichtzugehörigkeit ab. Jugendliche aus gehobenerem sozialem Umfeld befinden sich deutlich häufiger in Schulen mit höherem Anforderungsniveau. Der Schichteffekt fällt für den Schultyp sogar stärker aus als der Einfluss der kognitiven Grundfähigkeiten. Dagegen spielt die kulturelle Herkunft nach Kontrolle der sozialen Herkunft und der kognitiven Grundfähigkeiten - für die Zuteilung zu einem Schultyp keine Rolle. Daraus lässt sich folgern, dass Fremdsprachige bzw. immigrierte Jugendliche aus vergleichbaren sozialen Verhältnissen und bei gleichen intellektuellen Voraussetzungen hinsichtlich der Nutzung von Bildungsangeboten gegenüber Deutschsprachigen nicht benachteiligt sind. 


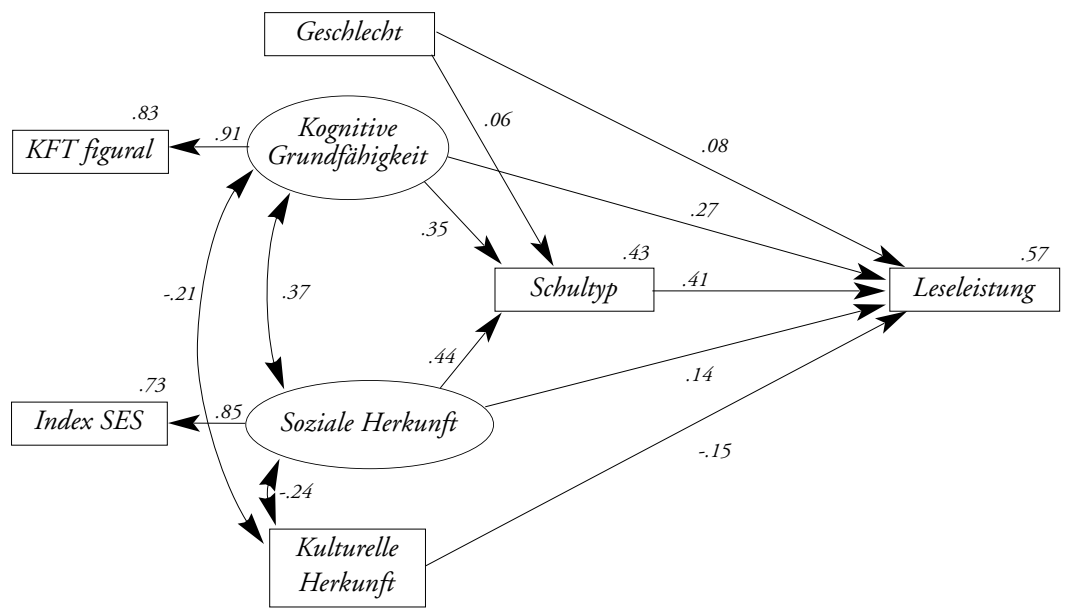

$\chi^{2}=1.845 \mathrm{df}=4 \quad p=.764 \mathrm{~g} f i=.999$ agf $i=.997$ rmsea $=.000$ pclose $=.995$

Abbildung 2: Erklärung der Leseleistung unter Einbezug kognitiver Grundfähigkeiten und des besuchten Schultyps $(N=1026)$

Um fachspezifische Unterschiede in den Zusammenhängen zu erkennen, wird in Abbildung 3 das gleiche Erklärungsmodell für mathematische Leistungen dargestellt. Die aufgenommenen Einflussgrössen erklären denselben Anteil an Varianz in den Mathematikleistungen wie beim Lesen. Der augenfälligste Unterschied zeigt sich bei der sozialen Herkunft, die keinen statistisch nachweisbaren, direkten Effekt auf mathematische Leistungen aufweist, sondern deren Einfluss über den Schultyp vermittelt wird. Dies kann als ein weiterer Hinweis für die grössere Schichtabhängigkeit von sprachlichen Kompetenzen interpretiert werden. Dagegen nimmt, verglichen mit dem Erklärungsmodell für das Lesen, der eigenständige Effekt der kognitiven Grundfähigkeiten zu, was zumindest teilweise damit begründet werden kann, dass der verwendete figurale Subtest des KFT keine Sprachfähigkeiten getestet hat. Unterschiede zeigen sich ausserdem beim Geschlecht: Die Knaben zeigen bessere Mathematikleistungen als die Mädchen und der ohnehin schwache Effekt zum Schultyp ist nicht mehr signifikant. 


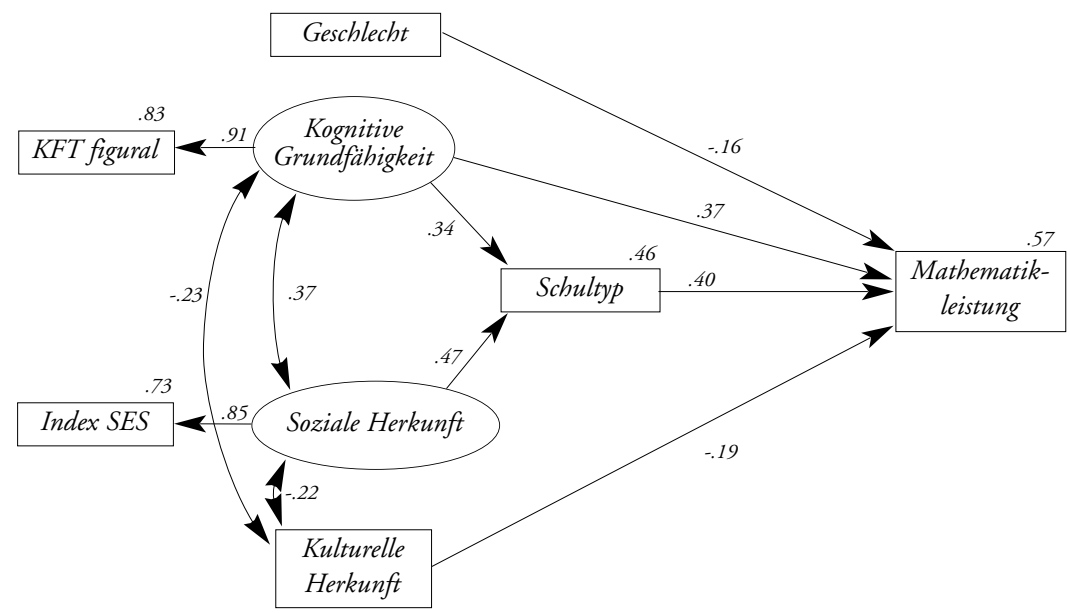

$\chi^{2}=8.260 \mathrm{df}=6 \quad p=.220 \mathrm{~g} f \mathrm{i}=.995$ agf $\mathrm{i}=.983$ rmsea $=.026$ plose $=.824$

Abbildung 3: Erklärung der Mathematikleistung unter Einbezug kognitiver Grundfähigkeiten und des besuchten Schultyps $(N=573)^{10}$

Anders als erwartet verhält sich der Zusammenhang zwischen der kulturellen Herkunft und der Mathematikleistung, der mit $\beta=-.19$ sogar ganz leicht grösser ausfällt als beim Lesen. Dieses Ergebnis widerspricht Moser und Rhyn (1999, S. 106 f.), die nach Kontrolle des Schultyps, der sozialen Herkunft, dem Geschlecht und der kognitiver Leistungsfähigkeit keinen Zusammenhang zwischen Fremdsprachigkeit und Mathematikleistung gefunden haben, während im Fachbereich Deutsch ein signifikanter Zusammenhang nachzuweisen war. Wenn allerdings die Leseleistung als Prädiktor für mathematische Leistungen hinzugefügt wird (vgl. Abbildung 4), so verringert sich der direkte Einfluss der kulturellen Herkunft auf die Mathematik.

Die Ergebnisse für ein (hier nicht abgebildetes) analoges Modell mit naturwissenschaftlicher Leistung als Kriterium sind jenen für das Lesen (vgl. Abbildung 2) sehr ähnlich. Die einzige nennenswerte Abweichung zeigt sich in der Umkehrung des Geschlechtereffekts $(\beta=-13)$.

Wenn Leseverständnis über den Fachbereich Deutsch hinaus als eine grundlegende Voraussetzung für den Lernerfolg angesehen wird, müsste die Lesekompetenz einen wesentlichen Beitrag für die Vorhersage mathematischer und naturwissenschaftlicher Grundbildung leisten. In einem weiteren Schritt wird deshalb die Leseleistung als Prädiktor für die mathematische Leistung in das Modell eingeführt (vgl. Abbildung 4). 


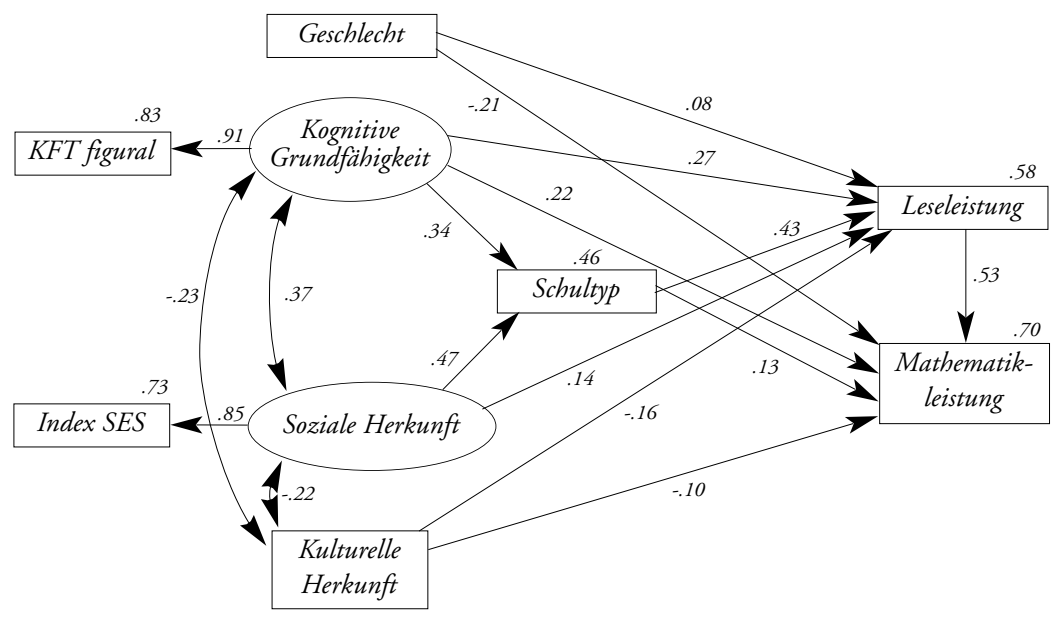

$$
\chi^{2}=6.205 d f=6 \quad p=.401 \quad g f i=.997 \text { agf } i=.986 \text { rmsea }=.008 \text { pclose }=.917
$$

\section{Abbildung 4: Erklärung der Mathematikleistung unter Einbezug kognitiver Grundfähigkeiten, mit Schultyp und Leseleistung als Prädiktoren $(N=573)$}

Mit dem Einfügen der Leseleistung wird ein erheblicher Teil der Unterschiede $(70 \%)$ in der Mathematikleistung erklärt. Wie erwartet ist der Einfluss der Lesekompetenz auf die Leistung in der Mathematik beachtlich $(\beta=.53)$. Ein Teil des Effekts der kognitiven Grundfähigkeit wirkt nun indirekt über die Leseleistung, wobei der direkte Pfad auf die Mathematik mit $\beta=.22$ stärker ist als der indirekte über die Leseleistung (.14). Demgegenüber ist beim Schultyp der indirekte Zusammenhang über die Leseleistung (.23) ausgeprägter als der direkte Pfad $(\beta=.13)$.

$\mathrm{Zu}$ erwähnen ist ferner, dass der direkte Pfad vom Geschlecht zur Mathematikleistung bei der gleichzeitigen Berücksichtigung der Leseleistung etwas stärker wird. Dies bedeutet, dass der Leistungsrückstand der Mädchen in der Mathematik bei gleichen Leseleistungen etwas deutlicher ausfällt. Der Zusammenhang zwischen der sozialen Herkunft und der Leistung wird nach wie vor hauptsächlich durch den Schultyp geprägt.

In einem analogen Modell mit naturwissenschaftlicher Leistung werden sogar 82 Prozent der Gesamtvarianz erklärt. Die Vorhersagekraft der Leseleistung auf die naturwissenschaftliche Leistung ist äusserst hoch $(\beta=.80)$. Der Nachweis guter naturwissenschaftlicher Leistungen setzt folglich fundierte Lesekompetenzen voraus. Dabei gilt es zu berücksichtigen, dass die naturwissenschaftlichen Testaufgaben stark textlastig waren und ohne ein genügendes Leseverständnis viele Aufgaben nicht lösbar waren. Dieser Befund unterstreicht die Notwendigkeit guter Lesefähigkeiten für erfolgreiches schulisches Lernen. 
Wie bei der Mathematik zeigt sich bei den Naturwissenschaften der Leistungsrückstand der Mädchen deutlicher, wenn die Leseleistung kontrolliert wird ( $\beta=-.21)$. In anderen Worten: Die Mädchen profitieren bei den naturwissenschaftlichen Aufgabenstellungen von ihren insgesamt besseren Lesefähigkeiten. Anders als beim Modell zur Mathematik verhält es sich bei der kulturellen Herkunft, die nur indirekt über das Lesen einen Einfluss auf die naturwissenschaftliche Leistung ausübt.

Diskussion

Sämtliche Analysen der PISA-Daten haben die grosse Bedeutung der sozialen Herkunft bzw. der Bildungsnähe des Elternhauses für fachliche Leistungen betont (z.B. BFS/EDK, 2002; OECD, 2001). Die vorliegende Analyse belegt, dass der Schichteffekt auch bei statistischer Kontrolle der kognitiven Grundfähigkeiten und der kulturellen Herkunft als ein eigenständiger Effekt nachzuweisen ist. Gleiches gilt - allerdings in deutlich geringerem Ausmass - für die kulturelle Herkunft. Der vergleichsweise geringe Effekt der kulturellen Herkunft deckt sich mit dem Befund von Moser und Rhyn (2000, S. 85), dass Fremdsprachigkeit für den Lernerfolg in Mathematik und Deutsch eine geringere Rolle spiele als die soziale Herkunft.

Der Schichteffekt wird in hohem Masse durch die Zugehörigkeit zu einem bestimmten Schultyp vermittelt. Die kulturelle Herkunft dagegen hat für das Absolvieren eines bestimmten Schultyps keine Bedeutung. Dieses Grundmuster zeigt sich in ähnlicher Weise sowohl beim Lesen als auch in der Mathematik und in den Naturwissenschaften. Der Schultyp erhält damit eine Schlüsselrolle für das Verständnis der Wirkung der sozialen Herkunft auf schulische Leistungen.

Nimmt man die Rolle der Lesekompetenz als eine Schlüsselqualifikation für weiteres Lernen ernst und betrachtet die Leseleistung als Prädiktor für die Leistung in Mathematik und Naturwissenschaften, so zeigen die Pfadmodelle einen starken Effekt der Leseleistung an. Dies gilt besonders für naturwissenschaftliche Leistungen. Durch diese Modellerweiterung wird zudem der auf Kosten der Frauen gehende Einfluss des Geschlechts auf die Leistungen in Mathematik und Naturwissenschaften deutlicher sichtbar. Aus der zentralen Stellung des Lesens für andere fachliche Kompetenzen lässt sich folgern, dass sich eine Förderung der Lesekompetenzen auch auf andere schulische Bereiche positiv auswirken würde.

\section{Chancenunterschiede beim übertritt ins Gymnasium und die Sekundarschule}

Die oben dargestellten Pfadmodelle haben in Übereinstimmung mit früheren Analysen (z.B. Ramseier, 1997) die zentrale Rolle gezeigt, die der Schultyp für die Erklärung des Zusammenhangs von sozialer Herkunft und Leistung hat. 
Diese Rolle soll im Folgenden anhand der relativen Chancen für den Besuch des Gymnasiums bzw. der Sekundarschule genauer analysiert werden. Entsprechend der einleitend formulierten zweiten Fragestellung geht es dabei um den Einfluss von sozialer und kultureller Herkunft sowie von Geschlecht, kognitiven Grundfähigkeiten und Leseleistungen. Der Zusammenhang dieser Faktoren mit den Übertrittschancen wird in drei Modellen zunehmend präziser gefasst. Im Nullmodell (M0, vgl. Tabellen 3 und 4) wird jeweils der bivariate Zusammenhang zwischen der Übertrittschance und den einzelnen Faktoren dargestellt, ohne Konfundierungen auszuschliessen. Im zweiten Modell M1 werden die schon vor Schulbeginn (weitgehend) festgelegten Faktoren in die Prognose des Übertritts einbezogen. Dieses Modell zeigt z.B. für die kulturelle Herkunft, in welchem Ausmass sie zur Übertrittsprognose beiträgt, wenn der Einfluss der übrigen Ausgangsmerkmale (soziale Herkunft, kognitive Grundfähigkeiten und Geschlecht) kontrolliert wird.

Im abschliessenden Modell M2 wird zusätzlich die Leseleistung in die Prognose einbezogen. Bei der sozialen Herkunft zeigt der so berechnete Koeffizient an, wie sich diese bei sonst gleichen Voraussetzungen (Grundfähigkeiten, Leistungen, Geschlecht, kulturelle Herkunft) auf die Übertrittschancen auswirkt. Dieses Modell erfasst somit die einleitend beschriebene sekundäre Chancenungleichheit. Dem Charakter der abhängigen Variablen entsprechend (Besuch ja/nein) werden die Modelle mit Hilfe der logistischen Regression berechnet.

\section{Übertritt ins Gymnasium}

Tabelle 3 zeigt die Ergebnisse der Modellberechnungen für den Übertritt ins Gymnasium. Für die Variablen bzw. Vergleichsgruppen (Prädiktoren) wird die relative Chance (odds ratio) aufgeführt. Diese gibt an, um welchen Faktor sich die Übertrittschancen ändern, wenn der Prädiktor um eine Einheit zunimmt. Es wird von Übertrittschancen (odds) und nicht von den vertrauteren Übertrittswahrscheinlichkeiten ausgegangen, da sich nur diese Chancen über unterschiedliche Situationen (Ausgangswahrscheinlichkeiten) hinweg einfach vergleichen lassen. Die Übertrittschance entspricht - ähnlich wie ein Wettquotient - dem Verhältnis der Wahrscheinlichkeit, den Übertritt zu schaffen, zur Wahrscheinlichkeit, den Übertritt nicht zu schaffen. Am Beispiel: Deutschsprechende treten gemäss unseren Daten zu 25\% ins Gymnasium über. Ihre Übertrittschance ist somit 25:75, also 1:3 bzw. ein Drittel. Die Übertrittschance der im Ausland geborenen Fremdsprachigen ist um den Faktor 0.41 kleiner (vgl. die entsprechende relative Chance in Tabelle 3) und beträgt rund 0.14, womit für sie die Wahrscheinlichkeit, ins Gymnasium überzutreten, gut $12 \%$ beträgt. Wie der p-Wert von .024 in Tabelle 3 zeigt, ist dies auf dem 5\%-Niveau statistisch signifikant niedriger als die Übertrittschance der Deutschsprachigen. Die Übertrittschance der in der Schweiz geborenen Fremdsprachigen ist dagegen nicht signifikant niedriger als jene der Deutschsprachigen. 
Tabelle 3: Relative Chancen zum Besuch des Gymnasiums

\begin{tabular}{|l|c|c|c|c|c|c|}
\hline & \multicolumn{2}{|l|}{ M0: Nullmodell } & \multicolumn{2}{c|}{$\begin{array}{c}\text { M1: Ohne Leis- } \\
\text { tungsvariablen }\end{array}$} & \multicolumn{2}{c|}{$\begin{array}{c}\text { M2: Mit Leis- } \\
\text { tungsvariablen }\end{array}$} \\
\hline Prädiktoren & R.C. & $\mathrm{p}$ & R.C. & $\mathrm{p}$ & R.C. & $\mathrm{p}$ \\
\hline Soziale Herkunft & 5.37 & $<.001$ & 4.56 & $<.001$ & 3.22 & $<.001$ \\
\hline Geschlecht & 1.13 & 0.410 & 1.19 & 0.152 & 0.92 & 0.660 \\
\hline Kulturelle Herkunft & & & & 0.912 & & 0.157 \\
\hline Fremdsprachig, in CH geboren & 0.74 & 0.316 & 0.95 & 0.842 & 1.72 & 0.163 \\
\hline Fremdsprachig, im Ausland geboren & 0.41 & 0.024 & 1.13 & 0.711 & 2.21 & 0.158 \\
\hline Kognitive Grundfähigkeit (figural) & 3.03 & $<.001$ & 2.39 & $<.001$ & 1.25 & 0.168 \\
\hline Kognitive Grundfähigkeit (verbal) & 5.54 & $<.001$ & & & 1.81 & 0.001 \\
\hline Leseleistung & 9.2 & $<.001$ & & & 4.74 & $<.001$ \\
\hline Modelle insgesamt: & & & $\mathrm{R}$ & & $\mathrm{R}^{2}$ & \\
\hline R2 (Nagelkerke) & & & 0.32 & & 0.45 & \\
\hline R2 (Nagelkerke) ohne soziale Herkunft & & & 0.15 & & 0.38 & \\
\hline
\end{tabular}

Anmerkungen: R.C.: Relative Chance (odds ratio); bei stetigen Variablen wurde auf den Vergleich zwischen 75. und 25. Perzentil im Prädiktor standardisiert. Das Geschlecht wurde als $0=$ Knaben und $1=$ Mädchen kodiert. Bei der kulturellen Herkunft wurden in zwei dummyVariablen die Deutschsprechenden mit 0, die Fremdsprachigen mit Geburtsort in der Schweiz bzw. im Ausland mit 1 codiert. Stichprobengrösse (halbiert): $N=1009$.

Damit die relativen Chancen für die stetig gemessenen Variablen wie die soziale Herkunft, die Leseleistung und die kognitiven Grundfähigkeiten vergleichbar sind, wurde die zugrunde gelegte Skala so normiert, dass eine Einheit dem Übergang vom 25. zum 75. Perzentil entspricht. Verglichen werden damit die Übertrittschancen von Kindern, deren soziale Herkunft (bzw. Leistung ...) von 25 Prozent aller Kinder unterschritten bzw. übertroffen wird. Die entsprechenden Koeffizienten des Nullmodells zeigen somit, dass der Übertritt ins Gymnasium ähnlich eng mit der sozialen Herkunft wie mit den kognitiven Grundfähigkeiten zusammenhängt, während die Lesekompetenz einen Übertritt weitaus am besten voraussagen lässt. Die der sozialen Herkunft zugeordnete relative Chance von 5.37 bedeutet gemäss dem zugrunde gelegten Regressionsmodell, dass die Übertrittswahrscheinlichkeit eines Kindes mit einer eher hohen sozialen Herkunft 42 Prozent beträgt, verglichen mit 12 Prozent für ein Kind mit eher niedriger sozialer Herkunft (75. verglichen mit 25. Perzentil).

Das Modell M1 dokumentiert einen immer noch starken Effekt der sozialen Herkunft auf die Übertrittschance ins Gymnasium, auch wenn nach figuralen 
kognitiven Grundfähigkeiten, kultureller Herkunft und Geschlecht kontrolliert wird. $\mathrm{Zu}$ diesen sozial ungleich verteilten Übertrittschancen können Leistungsunterschiede je nach sozialer Herkunft beitragen, die im Laufe der Jahre entstanden sind. Diese akkumulierten Leistungsunterschiede dürften hauptsächlich den nach sozialer Herkunft unterschiedlichen Lernumwelten zuzuschreiben sein, da ja nach den figuralen Grundfähigkeiten kontrolliert wird.

Das Ergebnis fällt für die kulturelle Herkunft verschieden aus. Wird nach sozialer Herkunft und figuraler kognitiver Grundfähigkeit kontrolliert, so hat die Fremdsprachigkeit, auch in Kombination mit Zuwanderung, keinen signifikanten Einfluss auf die Wahrscheinlichkeit eines Gymnasiumsbesuchs. Insgesamt erklären die Prädiktoren ohne soziale Herkunft den Gymnasiumsübertritt nur schlecht (Pseudo-R2 = .15). Unter Einbezug der sozialen Herkunft ist die Erklärungskraft zwar wesentlich höher, aber immer noch recht tief.

Selbst unter Berücksichtigung der Leseleistung (Modell M2) zeigt die soziale Herkunft einen starken Effekt auf die Chance eines Gymnasiumsbesuchs. Die an die soziale Herkunft geknüpfte relative Chance von 3.22 bedeutet, dass wenn ein Schüler bzw. eine Schülerin mit relativ hoher sozialer Herkunft (75. Perzentil) bei einer bestimmten mittleren Konstellation von Leseleistung und kognitiven Grundfähigkeiten ${ }^{11}$ mit 36\% Wahrscheinlichkeit ins Gymnasium übertritt, ein Kind bei gleicher Konstellation jedoch mit relativ niedriger sozialer Herkunft (25. Perzentil) dies nur mit 15 Prozent Wahrscheinlichkeit tut. Auch der zusätzliche Einbezug der Leistung in Mathematik bzw. Naturwissenschaften ändert an diesem Effekt der sozialen Herkunft praktisch nichts. Diese sekundäre Chancenungleichheit, die bei gleichen kognitiven Fähigkeiten und gleichen Leistungen festzustellen ist, ist damit beim Übertritt ins Gymnasium hoch.

Während die kognitiven Grundfähigkeiten und die Leseleistung wie zu erwarten signifikant zur Prognose des Gymnasiumsübertritts beitragen, tun dies das Geschlecht und die kulturelle Herkunft nicht. Bei gleichen Leseleistungen und gleicher sozialer Herkunft zeichnet sich gar ein schwacher, nicht signifikanter Trend in die Gegenrichtung ab: Mädchen und Deutschsprechende treten bei gleichen Leistungen und kognitiven Grundfähigkeiten etwas seltener ins Gymnasium über. Letzteres haben auch Baumert und Schümer (2002, S. 172) festgestellt.

\section{übertritt in die Sekundarschule}

Tabelle 4 beschreibt die Übertrittschancen in die Sekundarschule. Dabei wird der Besuch der Sekundarschule oder des Gymnasiums in der 9. Klasse dem der Realschule gegenübergestellt.

In groben Zügen ist das Bild ähnlich wie beim Übertritt ins Gymnasium: Soziale Herkunft und Übertrittschance hängen, ohne Kontrolle weiterer Faktoren, eng zusammen; ein Zusammenhang, der auch nach der Kontrolle der kognitiven Grundfähigkeiten (M1) und zusätzlich der Leseleistung (M2) festzustellen ist. Eine zusätzliche Analyse zeigt, dass auch der Einbezug der Leistung in Mathe- 
matik oder Naturwissenschaften den Effekt der sozialen Herkunft nicht senkt. Wie beim Gymnasiumsübertritt liegt ein klares Indiz für sekundäre Chancenungleichheit vor.

Tabelle 4: Relative Chancen zum Besuch der Sekundarschule oder des Gymnasiums

\begin{tabular}{|c|c|c|c|c|c|c|}
\hline & \multicolumn{2}{|c|}{ M0: Nullmodell } & \multicolumn{2}{|c|}{$\begin{array}{l}\text { M1: Ohne Leis- } \\
\text { tungsvariablen }\end{array}$} & \multicolumn{2}{|c|}{$\begin{array}{l}\text { M2: Mit Leis- } \\
\text { tungsvariablen }\end{array}$} \\
\hline Prädiktoren & R.C. & $\mathrm{p}$ & R.C. & $\mathrm{p}$ & R.C. & $\mathrm{p}$ \\
\hline Soziale Herkunft & 4.65 & $<.001$ & 3.33 & $<.001$ & 1.84 & 0.001 \\
\hline Geschlecht & 1.50 & 0.004 & 1.68 & 0.002 & 1.22 & 0.329 \\
\hline Kulturelle Herkunft & & & & 0.013 & & 0.229 \\
\hline Fremdsprachig, in $\mathrm{CH}$ geboren & 0.59 & 0.034 & 0.84 & 0.566 & 1.88 & 0.088 \\
\hline Fremdsprachig, im Ausland geboren & 0.17 & $<.001$ & 0.36 & 0.003 & 1.01 & 0.989 \\
\hline Kognitive Grundfähigkeit (figural) & 3.85 & $<.001$ & 3.32 & $<.001$ & 1.62 & $<.001$ \\
\hline Kognitive Grundfähigkeit (verbal) & 9.72 & $<.001$ & & & 2.47 & $<.001$ \\
\hline Leseleistung & 23.6 & $<.001$ & & & 9.75 & $<.001$ \\
\hline Modelle insgesamt: & & & $\mathrm{R}^{2}$ & & $\mathrm{R}^{2}$ & \\
\hline $\mathrm{R}^{2}$ (Nagelkerke) & & & 0.41 & & 0.62 & \\
\hline $\mathrm{R}^{2}$ (Nagelkerke) ohne soziale Herkunft & & & 0.33 & & 0.61 & \\
\hline
\end{tabular}

Anmerkungen: R.C.: Relative Chance (odds ratio); bei stetigen Variablen wurde auf den Vergleich zwischen 75. und 25.Perzentil im Prädiktor standardisiert. Das Geschlecht wurde als $0=$ Knaben und 1 = Mädchen kodiert. Bei der kulturellen Herkunft wurden in zwei dummyVariablen die Deutschsprechenden mit 0, die Fremdsprachigen mit Geburtsort in der Schweiz bzw. im Ausland mit 1 codiert. Stichprobengrösse (halbiert): $N=1009$.

Im abschliessenden Modell M2 wird die Leseleistung als ein Prädiktor zur Selektion in die verschiedenen Schultypen betrachtet, obwohl die Leistung ja im 9. Schuljahr, also nach dem Übertritt, gemessen wurde. Der einleitend beschriebene Scheren-Effekt hat zur Folge, dass sich in den typenspezifischen Milieus die zum Selektionszeitpunkt bestehenden Leistungsunterschiede vergrössern. Die hier nachträglich gemessenen Leistungen überschätzen damit den Einfluss der Leistungsunterschiede auf den Übertritt. Ein Teil der Effekte, die beim Übertritt sozial bedingt waren, setzen sich bis zum 9. Schuljahr in Leistungsunterschiede um und werden im Modell M2 statt der sozialen Herkunft der Leistung zugeschrieben. Als Preis für die Annäherung an eine Frage, die eigentlich nur mit 
längsschnittlichen Daten genau untersucht werden kann, wird folglich die Bedeutung der sozialen Herkunft unterschätzt. Diese Unterschätzung dürfte beim Übertritt in die Sekundarschule erheblicher sein als bei jenem ins Gymnasium, da sich der Schereneffekt in der Sekundarschule bis zum Testzeitpunkt während fast dreier Jahre, beim Gymnasium dagegen nur während knapp eines Schuljahres entfalten kann.

Das Nullmodell zeigt, dass Knaben und Fremdsprachige signifikant kleinere Chancen haben, die Sekundarschule zu besuchen als Mädchen bzw. Deutschsprechende. Die kleineren Übertrittschancen nähern sich jedoch jenen der Vergleichsgruppen an, wenn man Herkunft, Grundfähigkeiten und erst recht die Leseleistung kontrolliert. Bei vollständiger Kontrolle zeigt sich sogar ein geringer, aber nicht signifikanter Trend, dass fremdsprachige in der Schweiz geborene Jugendliche bei gleichen Leseleistungen und kognitiven Grundfähigkeiten etwas häufiger die Sekundarschule besuchen als deutschsprechende. Die nähere Analyse zeigt, dass die in der Schweiz geborenen Fremdsprachigen, die in die Sekundarschule kommen, nicht die typischen Vertreter der zweiten Generation von Einwanderern sind. Vielmehr sind viele von ihnen Französischsprechende aus dem Kanton Bern oder Jugendliche, von denen auch der Vater oder die Mutter in der Schweiz geboren sind.

\section{Diskussion}

Die bereits von Zutavern et al. (2002) festgestellten sozialen Ungleichheiten im Zugang zu Sekundarschulen und Gymnasien werden durch die Kontrolle von kognitiven Grundfähigkeiten, kultureller Herkunft und Fachleistungen erhärtet und präzisiert. Vor allem beim Gymnasium ist eine erhebliche sekundäre Chancenungleichheit festzustellen: Jugendliche mit niedriger sozialer Herkunft gelangen bei gleichen Kompetenzen seltener ins Gymnasium als Jugendliche mit hoher sozialer Herkunft. Wie Baumert und Schümer (2002) gehen wir davon aus, dass dabei das Motiv des Statuserhalts zwischen Generationen, unterschiedliche Bildungsaspirationen und Bereitschaften zu Investitionen in weiterführende Ausbildungen ausschlaggebend sind. Aber auch ein vom sozialen Status der Schülerinnen und Schüler abhängiges Verhalten der Schulen bei der Beratung und der Abgabe von Empfehlungen kann eine Rolle spielen. Dass diese sekundäre soziale Chancenungleichheit beim Übertritt in die Sekundarschule weniger ausgeprägt ist, ist wohl eine Folge davon, dass mit der allgemeinen Bildungsexpansion die Sekundarschule für alle sozialen Schichten in Reichweite liegt. Ähnlich wie in Deutschland liegt die soziale Wasserscheide der Bildungsbeteiligung damit zunehmend zwischen dem Besuch eines Gymnasiums und einer Sekundarschule (Baumert \& Schümer, 2001; Lamprecht \& Stamm, 1996). Aus bildungspolitischer Sicht ist wieder einmal das Fazit zu ziehen, dass bei den stark selektierenden Schulstrukturen der Sekundarstufe I und den damit verbundenen Übertrittsentscheiden ein wichtiger Ansatzpunkt für eine Reduktion der in der Schweiz engen Koppelung von sozialer Herkunft und Fachleistungen zu suchen ist. 
Nach Kontrolle von kognitiven Grundfähigkeiten, sozialer Herkunft und Leseleistung ist dagegen die kulturelle Herkunft für die Chance des Besuchs von Gymnasium oder Sekundarschule nicht relevant. Dabei sind sowohl Sprachhintergrund als auch Immigrationsstatus unerheblich. Es kann gefolgert werden, dass das Schulsystem hinsichtlich der kulturellen Herkunft nicht diskriminierend wirkt. Dass Fremdsprachige bei gleichen Leistungen tendenziell sogar vermehrt in einen weiterführenden Schultyp gelangen, kann daran liegen, dass es besondere Kompetenzen bedingt, um beim Lesen in einer Fremdsprache auf die gleiche Leistung zu kommen wie die andern beim Lesen in der Muttersprache. Diese hier nicht vollständig erfassten zusätzlichen Kompetenzen können dann auch zur Aufnahme in die weiterführende Schule führen.

Die sekundäre Chancenungleichheit ist der offensichtlichste Beleg für die soziale Selektivität des Bildungssystems. Doch der Prozess beginnt schon früher und es können sich Leistungsunterschiede akkumulieren, die hauptsächlich den nach sozialer Herkunft unterschiedlichen Lernumwelten zugeschrieben werden können. Dementsprechend haben Moser, Keller und Tresch (2002) denn auch bereits am Ende der dritten Primarschulklasse eine grosse soziale Selektivität festgestellt. Dieser zusätzliche Einfluss wird hier anhand der höheren Ungleichheit der Übertrittschancen deutlich, wenn nicht auf Fachleistungen, wohl aber auf nicht-verbale kognitive Grundfähigkeiten, kulturelle Herkunft und Geschlecht kontrolliert wird. Damit wird eine globale Chancenungleichheit beschrieben, die sich aus der sozial unterschiedlichen Entwicklung (inklusive den Auswirkungen früherer Entscheide über die Teilnahme am Bildungssystem) und den Ungleichheiten beim fraglichen Übertritt ergeben. Neben der Art der Weichenstellung in der Sekundarstufe I ist deshalb eine frühzeitige, sozial ausgeglichene kognitive Förderung notwendig, wenn die Verknüpfung von Herkunft und Leistung gelockert werden soll. Die Einführung einer Basisstufe zur Neugestaltung des Schuleintritts (EDK, 1997) könnte dazu einen wichtigen Beitrag leisten.

\section{Einfluss der einzelnen Schule auf die Leseleistung}

Im Folgenden werden Schuleffekte untersucht. Der Begriff «Schuleffekt» verweist auf unterschiedliche Sachverhalte (vgl. Scheerens \& Bosker, 1997). Um die Bedeutung der einzelnen Schule für das Entstehen von Leistungsunterschieden zu verdeutlichen, wird eine zunehmend engere Definition des Schuleffekts verwendet. Im ersten Abschnitt wird die Grösse von Schuleffekten bestimmt; im zweiten und dritten Abschnitt werden Schulmerkmale identifiziert, die zu diesen Effekten beitragen. 
Leistungsunterschiede zwischen und innerhalb von Schulen Der Brutto-Schuleffekt bezeichnet den Unterschied zwischen der Durchschnittsleistung einer einzelnen Schule und dem Gesamtdurchschnitt aller Schulen. Um den Brutto-Effekt in der Gesamtheit der Schulen zu beschreiben, kann er als Verhältnis der Leistungsvarianz zwischen Schulen zur Gesamtvarianz beschrieben werden (Intraklassenkorrelationskoeffizient). Bei den neunten Klassen der Kantone Bern und St. Gallen beträgt der Brutto-Schuleffekt 37 Prozent (vgl. Abbildung 5, linker Teil). ${ }^{12}$

Brutto-Schuleffekte dürfen jedoch nicht allein als Wirkung der einzelnen Schulen angesehen werden. Sie hängen ebenso von den unterschiedlichen Lernvoraussetzungen $a b$, über die ihre Schülerinnen und Schüler beim Eintritt in diese Schule verfügen. Als Mass für die Wirkung der einzelnen Schulen bzw. für Wirkungsunterschiede werden deshalb Netto-Schuleffekte berechnet, indem Unterschiede in den individuellen Voraussetzungen der Schülerinnen und Schüler wie ihre kognitiven Grundfähigkeiten, ihre soziale und kulturelle Herkunft und ihr Vorwissen kontrolliert werden. Netto-Schuleffekte sind Abweichungen in der Leistung einer Schule von dem, was man aufgrund der individuellen Voraussetzungen ihrer Schülerinnen und Schüler erwarten würde (Willms, 1992). In einem nach Schultypen gegliederten Schulsystem muss zudem der Schultyp möglichst genau einbezogen werden. Auf Grund des Selektionsprozesses bestehen zwischen den Typen grosse Leistungsunterschiede, die nicht der einzelnen Schule zugeschrieben werden dürfen.

Abbildung 5 gibt im rechten Teil einen Überblick über die unterschiedlichen Varianzanteile. Diese wurden geschätzt, indem die Varianzkomponenten innerhalb und zwischen Schulen zuerst ohne feste Faktoren, dann mit Einbezug der erfassten Individualmerkmale, des Schultyps sowie deren Kombination geschätzt wurden. Der Vergleich der differenzierten Aufteilung mit dem Brutto-Schuleffekt (linker Teil der Abbildung) zeigt, wie wenig letzterer aussagt. Dazu trägt bei, dass sich der Schultyp in den Kantonen Bern und St. Gallen teils auf der Schulebene, teils innerhalb der Schule auswirkt: In St. Gallen wird der ganze, in Bern ein Teil des gymnasialen Unterrichts an separaten Gymnasien angeboten. Realschulen werden in beiden Kantonen teilweise als eigenständige Schulen, teils innerhalb eines Oberstufenzentrums zusammen mit Sekundarschulen geführt. Der Brutto-Effekt spiegelt unter anderem dieses Mischungsverhältnis und würde für konsequent typengetrennte bzw. typenintegrierte Systeme anders aussehen. Unterschiede in der Varianzaufteilung zwischen den beiden Kantonen sind im Übrigen klein und liegen im Bereich des Schätzfehlers, weshalb die Ergebnisse auch nicht nach den Kantonen getrennt werden. 


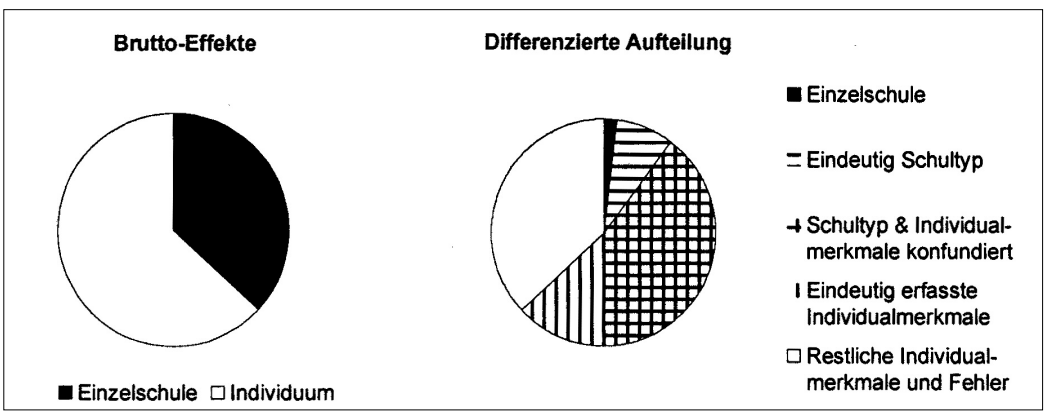

Abbildung 5: Schuleffekte im Rahmen unterschiedlich differenzierter
Varianzaufteilung

Wie sehr Leistungsunterschiede zwischen Schultypen auf Selektionsprozesse zurückzuführen sind, zeigt sich am grossen Varianzanteil (39\%), der weder eindeutig dem Schultyp noch den erfassten Individualmerkmalen zugeschrieben werden kann. Auch zum Varianzanteil, der eindeutig dem Schultyp zugeschrieben wird (8\%), können nicht erfasste, aber selektionswirksame individuelle Unterschiede beitragen. Dieser Varianzanteil erfasst damit nicht genau die Wirkung der einleitend beschriebenen typenspezifischen Lernumgebungen, sondern stellt dafür eine Obergrenze dar. Wie bedeutsam die erfassten Individualmerkmale der kognitiven Grundfähigkeiten ${ }^{13}$, des Geschlechts sowie der sozialen und kulturellen Herkunft für die Erklärung von Leistungsunterschieden im Lesen sind, zeigt sich u. a. am eindeutig auf die erfassten Merkmale zurückzuführenden Varianzanteil (13\%). Wenn man bedenkt, dass viele weitere individuelle Faktoren und nicht zuletzt die Messfehler die verbleibende individuelle ${ }^{14}$ Varianz bestimmen, mutet sie mit 37\% eher bescheiden an.

Von besonderem Interesse ist der nach all diesen Kontrollen den einzelnen Schulen zuschreibbare Varianzanteil (2\%). Er entspricht einem Anteil von 5\% an der nicht durch die Kontrollfaktoren erklärten Varianz. Dieser Netto-Schuleffekt von 5\% ist zwar eher klein; er liegt aber in einer üblichen Grössenordnung (Scheerens \& Bosker, 1997) und ist statistisch gesichert ( $c 2=152.6$; $\mathrm{df}=60$; $\mathrm{p}<.001)$. Damit können Leistungsunterschiede nachgewiesen werden, die als Wirkung der einzelnen Schulen anzusehen sind.

Willms (1992) und Scheerens und Bosker (1997) bezeichnen die bisher dargestellten, auf die individuellen Voraussetzungen korrigierten Netto-Effekte als Effekte vom Typus A. Effekte vom Typus B sind dagegen zusätzlich auf Unterschiede korrigiert, die sich aus der kollektiven Zusammensetzung einer Schule ergeben (vgl. nächster Abschnitt). Unter Kontrolle der kollektiven Wirkung von sozialer und kultureller Herkunft entspricht der Netto-Schuleffekt vom Typ B hier einem Anteil von 4\% erklärter Varianz $\left(\chi^{2}=133.3\right.$; $\mathrm{df}=58$; $\left.\mathrm{p}<.001\right)$. Dieser Netto-Effekt vom Typus B kommt jenem Schuleffekt am nächsten, der die 
Wirkung («value added») aller von Schule zu Schule unterschiedlichen pädagogischen und organisatorischen Praktiken wie Unterrichtsmethode, Schulklima, Lehrerqualifikation oder Schulgrösse repräsentiert.

\section{Effekte der Herkunft auf Individual- und Schulebene}

Wenn Schulen sich in ihrer sozialen Zusammensetzung oder im Prozentsatz fremdsprachiger Schülerinnen und Schüler stark unterscheiden, kann dieses Schulmerkmal zu unterschiedlichen Lernbedingungen führen und so Auswirkungen auf die Leistung eines Schülers bzw. einer Schülerin haben. Diese Auswirkungen sind unabhängig von der eigenen sozialen Herkunft und Sprachzugehörigkeit des Schülers bzw. der Schülerin. In der mehrebenenanalytischen Auswertung werden der Einfluss der Herkunft auf der Ebene des Individuums (Auswirkung der eigenen Herkunft) und der Schule (Einfluss der Zusammensetzung der Schule) gleichzeitig berechnet und können so verglichen werden.

Um die Wirkung der Zusammensetzung der Schülerschaft möglichst präzise zu erfassen, werden jeweils der Schultyp, die figuralen und verbalen Grundfähigkeiten, das Geschlecht sowie die persönliche kulturelle und soziale Herkunft auf Individualebene kontrolliert. Die angegebenen Effekte sind bei der sozialen Herkunft so standardisiert, dass sie die Leistungsveränderungen auf der PISALeseleistungsskala angeben, die einer Differenz von einer Standardabweichung ${ }^{15}$ in der sozialen Herkunft des Schülers oder der Schülerin oder aber im Mittelwert der Schule entspricht. Die kulturelle Herkunft wird auf Schulebene anhand des Anteils fremdsprachiger Schülerinnen und Schüler, ungeachtet ihres Geburtsorts, erfasst. Der angegebene Effekt entspricht einer Änderung dieses Anteils um 20 Prozentpunkte - ein grosser, aber realistischer Unterschied zwischen Schulen. Auf Individualebene geben die Effekte an, um wieviel sich im Ausland bzw. in der Schweiz geborene Fremdsprachige nach Kontrolle der übrigen Merkmale in der Leseleistung von Deutschsprechenden unterscheiden.

Tabelle 5 zeigt, dass die soziale Zusammensetzung der Schulen einen statistisch knapp signifikanten Effekt auf die Leistung hat. Der kollektive Effekt ist nominell fast doppelt so gross wie der individuelle. Seine Bedeutung sollte aber nicht überschätzt werden, denn nach Kontrolle des Schultyps beträgt der mittlere Unterschied der sozialen Zusammensetzung (SD) zwischen Schulen nur noch rund $18 \%$ der vollen Standardabweichung der individuellen sozialen Herkunft. Die faktisch auftretenden, kontrollierten Schulunterschiede aufgrund der sozialen Zusammensetzung entsprechen damit im Mittel nur etwa 2 bis 3 Punkten auf der Skala der Leseleistung.

Auch der Anteil Fremdsprachiger in einer Schule hat einen statistisch knapp signifikanten Effekt. Dieser weist auf erschwerte Lernbedingungen in Schulen hin, die einen hohen Anteil an Fremdsprachigen haben. Einen solchen Effekt haben auch Moser und Rhyn (2000, S. 98 ff.) festgestellt, wobei sie zeigen konnten, dass diese Wirkung erst erheblich wird, wenn im Anteil Fremdsprachiger eine Schwelle von etwa 40-50 Prozent überschritten wurde und dass sie vor al- 
lem Fremdsprachige betrifft (Moser \& Rhyn, 2000, S. 104; 1997, S. 32). Stanat (2002) und Baumert et al. (2003) haben einen solchen Schwelleneffekt in Deutschland bei einem rund 20-prozentigen Anteil Fremdsprachiger festgestellt.

Die Effekte der kulturellen und der sozialen Zusammensetzung sind teilweise konfundiert. Berechnet man sie unter zusätzlicher gegenseitiger Kontrolle, so sind sie einzeln nicht mehr signifikant. Die Stichprobe von 61 Schulen genügt eben nicht, um auf Schulebene zwischen den beiden Effekten sauber zu differenzieren. Dadurch wird aber der Hinweis auf ein Vorliegen der beiden Effekte nicht entkräftet. Die kleine Stichprobe erlaubt auch nicht, im Detail zu untersuchen, ob und wo ein Schwellenwert festgestellt werden kann, ab dem der Anteil Fremdsprachiger zu einer gewissen Minderung des Leistungsniveaus führt und ob eher die Deutschsprechenden oder die Fremdsprachigen betroffen sind.

Tabelle 5: Leseleistungen auf Schul- und Individualebene nach sozialer und kultureller Herkunft sowie nach Zusammengehörigkeitsgefühl

\begin{tabular}{lccc}
\hline Unabhängige Variablen & Effekt & SE & p-Wert \\
\hline $\begin{array}{l}\text { Soziale Herkunft } \\
\text { soziale Herkunft, Schulebene }\end{array}$ & 13.6 & 6.6 & 0.044 \\
$\quad$ soziale Herkunft, Individualebene & 7.7 & 1.8 & 0.000 \\
Kulturelle Herkunft & & & \\
$\quad$ Anteil Fremdsprachige & -7.0 & 3.4 & 0.043 \\
$\quad$ Fremdsprachig, in CH geboren & -40.6 & 7.6 & 0.000 \\
$\quad$ Fremdsprachig, im Ausland geboren & -24.6 & 5.6 & 0.000 \\
Zugehörigkeitsgefühl & & & \\
Zugehörigkeitsgefühl, Schulebene & 15.8 & 7.0 & 0.028 \\
Zugehörigkeitsgefühl, Individualebene & 2.0 & 1.4 & 0.169 \\
\hline
\end{tabular}

Anmerkungen: In diesen drei Auswertungen werden jeweils nur der Achsenabschnitt sowie der individuelle Fehler als Zufallsfaktor modelliert (Random Intercept Modelle). Der Schulyp wird als fester Faktor einbezogen, wobei das Gymnasium und die Sekundarschule in den Kantonen Bern bzw. St. Gallen je als separate Gruppen behandelt werden. Im Kanton Bern wird zudem der gymnasiale Unterricht an Oberstufenzentren (Schulen mit Einschluss von Realschulen) separat modelliert, da dort die Leistungen etwas niedriger sind als an reinen Gymnasien. Weitere feste Faktoren und die Normierung der Effekte werden im Text erwähnt.

Interessant ist der Vergleich der so bestimmten individuellen und kollektiven Effekte der sozialen Herkunft mit entsprechenden Effekten, die man erhält, wenn man nicht nach Schultyp und Individualmerkmalen kontrolliert. Die gleich standardisierten Effekte betragen dann auf Individualebene 24 und auf Schulebene volle 67 Punkte. Der Schuleffekt ist etwa fünfmal grösser als bei der oben vorgenommenen, kontrollierten Berechnung. Die Effekte sind damit in den 
neunten Klassen der beiden Kantone ähnlich gross wie jene bei den 15-Jährigen der ganzen Schweiz (24 Punkte individuell, 64 Punkte kollektiv; vgl. OECD, $2001,355)^{16}$. Es wäre völlig falsch, solche nicht auf den Schultyp kontrollierte Effekte, wie sie im ersten internationalen Bericht zu PISA verwendet werden, als kausale Wirkung der sozialen Segregation und der damit einhergehenden unterschiedlichen Lernbedingungen in den Schulen zu interpretieren: Der mittlere soziale Status der Schule hängt ja eng mit dem Schultyp zusammen. Wenn Schülerinnen und Schüler in einem gegliederten Schulsystem eine Schule mit hohem durchschnittlichem sozialem Status besuchen, ist das ein Indikator dafür, dass sie zum Selektionszeitpunkt gute individuelle Leistungen gezeigt haben und entsprechend in ein Gymnasium gelangt sind. Manche (wenn auch nicht alle) hochbegabten Kinder der Unterschicht gelangen ins Gymnasium, also in eine Schule mit hohem sozialen Status; manche (wenn auch nicht alle) Kinder der Oberschicht, die Lernschwierigkeiten haben, gelangen in die Realschule mit ihrem niedrigen mittleren sozialen Status. In beiden Fällen ist der soziale Status der besuchten Schule der weitaus bessere Prädiktor der Leistung als der eigene soziale Status. In gegliederten Schulsystemen überschätzt somit der nicht nach Schultyp kontrollierte kollektive Effekt der sozialen Herkunft die Wirkung der sozialen Segregation massiv.

\section{Effekte einzelner schulischer oder unterrichtlicher Merkmale}

Die oben (Abschnitt Leistungsunterschiede zwischen und innerhalb von Schulen) diskutierten Netto-Effekte auf Schulebene zeigen nur, dass Schulen sich voneinander im Leistungsniveau auch bei gleichem Schultyp und gleichen individuellen Voraussetzungen in der Schülerschaft unterscheiden. Ein Teil dieser Unterschiede wurde eben (Abschnitt Effekte der Herkunft auf Individual- und Schulebene) auf kollektive Effekte der sozialen bzw. kulturellen Zusammensetzung zurückgeführt. PISA hat einige schulische Merkmale erfasst, die gemäss den Ergebnissen der Schulwirksamkeitsforschung für den Lernerfolg relevant sind. Es fragt sich nun, ob für diese Merkmale Zusammenhänge mit der Leistung nachgewiesen werden können. Die hier vorgenommene Abklärung beschränkt sich auf Merkmale, die über den Schülerfragebogen erfasst wurden, die also die Wahrnehmung der Schülerinnen und Schüler repräsentieren. Zu den so erfassten Merkmalen gehören die Schuldisziplin, die Unterstützung durch die Lehrkräfte, die Qualität des Schüler-Lehrer-Verhältnisses, der Leistungsdruck, der Absentismus, das Zugehörigkeitsgefühl (sense of belonging) und der Zeitaufwand für Hausaufgaben.17 Diese Merkmale wurden unter Kontrolle der Individualmerkmale und des Schultyps auf Schul- und Individualebene eingeführt. Mit einer Ausnahme zeigen sich keine signifikanten Zusammenhänge. Dies ist nicht weiter erstaunlich, denn das Design von PISA, das eine über viele Jahre entwickelte Kompetenz mit den Lernbedingungen im Testjahr vergleicht, ist für eine solche Analyse wenig geeignet. 
Eine Ausnahme liefert das Zugehörigkeitsgefühl. In dieser Variablen werden die Reaktionen auf Aussagen wie "Meine Schule ist ein Ort an dem ... ich mich dazugehörig finde; ... ich leicht Freunde finde» zusammengefasst. Es zeigt sich, dass der Effekt dieser Variablen auf Schulebene, nicht aber auf Individualebene statistisch signifikant ist (siehe Tabelle 5). Das heisst, dass nicht die Befindlichkeit des Einzelnen im Zusammenhang mit seiner Leistung steht, sondern die Situation in der Schule, so wie sie von der Gesamtheit der Schülerinnen und Schüler eingeschätzt wird. Diese kollektive Einschätzung könnte das vorhandene soziale Klima besser erfassen als die Einzelurteile. Dass das Merkmal auf Schulebene einen signifikanten Effekt zeigt, könnte aber auch eine methodische Folge einer niedrigen Reliabilität auf Individualebene sein (Lüdtke, Robitzsch \& Köller, 2002).

\section{Diskussion}

Wie die Schuleffektivitätsforschung zeigt (Scheerens \& Bosker, 1997), muss der Einfluss der einzelnen Schule einbezogen werden, um die Wirkungsweise des Bildungssystems zu verstehen. Brutto- und Netto-Schuleffekte beschreiben, in welchem Ausmass die Schulen zur gesamten Leistungsvarianz beitragen; die Effekte bestimmter Schulmerkmale zeigen, worauf dieser Beitrag beruht. Im Abschnitt Leistungsunterschiede zwischen und innerhalb von Schulen wird der BruttoEffekt der Schulen einer differenzierten Aufgliederung gegenübergestellt, die den Schultyp und individuelle Merkmale berücksichtigt (Netto-Schuleffekt). Dieser Vergleich zeigt erneut die grosse Bedeutung des Schultyps, die zu einem grossen Teil mit selektionswirksamen Individualmerkmalen konfundiert ist. Nur Netto-Effekte nach Kontrolle von Schultyp und Individualmerkmalen dürfen als Wirkung der Schule interpretiert werden. Zur Situationsbeschreibung sind sowohl die Werte mit und ohne Ausklammern des Schultyps informativ, denn es wäre irreführend, die auf den Schultyp zurückzuführende Varianz verschwinden zu lassen. Um aber Schuleffekte sinnvoll interpretieren und vergleichen zu können, ist es in Ländern mit gegliedertem Schulsystem unerlässlich, dass auf der Sekundarstufe I der Schultyp detailliert erfasst und nicht wie bei PISA 2000 auf die ISCED-Klassifikation reduziert wird (Adams \& Wu, 2002).

Die detaillierte Varianzaufteilung zeigt, dass die einzelnen Schulen auch nach Kontrolle von Schultyp und individuellen Schülermerkmalen einen geringen aber statistisch signifikanten Effekt auf die Leistung haben. Auch wenn offen bleiben muss, wie weit sich hinter diesen Schuleffekten aggregierte Klasseneffekte verbergen (Scheerens \& Bosker, 1997), liegt damit ein Indiz vor, dass Unterschiede in den Praktiken und Eigenschaften der einzelnen Schulen bzw. Klassen eine Wirkung haben. Die Analyse oben hat gezeigt, dass insbesondere die Zusammensetzung der Schule nach sozialer und nach kultureller Herkunft ihrer Schülerschaft solche kollektive Effekte hat, die unabhängig von der Herkunft des einzelnen Schülers und der einzelnen Schülerin sind. Diese Effekte der Zusammensetzung nach Herkunft stellen eine grosse Herausforderung an die pädagogische Praxis dar. Nimmt sie die Forderung nach Chancengleichheit ernst, muss 
sie darauf abzielen, solche kollektiven Effekte zu vermeiden, denn diese führen für die Einzelnen zu ungleichen Lernmöglichkeiten.

Der festgestellte Effekt der sozialen Zusammensetzung der Schule ist bei Kontrolle von Schultyp und individuellen Merkmalen viel geringer als ohne Kontrolle. Vergleiche zwischen Ländern ohne diese Kontrolle (OECD, 2001, S. 355) führen somit in die Irre, wenn die Effekte der sozialen Zusammensetzung als Wirkungen interpretiert werden. Auch hier ist in gegliederten Schulsystemen eine exakte Erfassung des Schultyps unerlässlich. Dies darf aber umgekehrt nicht von der im Kapitel festgestellten Schlüsselrolle des Schultyps bei der Entstehung sozial bedingter Leistungsunterschiede ablenken.

Beim Design von PISA erstaunt es nicht, dass es kaum gelingt, die Wirkung konkreter Praktiken von Schule und Unterricht nachzuweisen. Von mehreren untersuchten Merkmalen zeigte nur eines einen statistisch signifikanten Effekt: Schulen, denen es besser gelingt, ihrer Schülerschaft ein hohes Zugehörigkeitsgefühl zu vermitteln, erreichen auch ein höheres Leistungsniveau (Abschnitt $E f$ fekte einzelner schulischer oder unterrichtlicher Merkmale). Dass gerade das $\mathrm{Zu}-$ gehörigkeitsgefühl als ein relevantes Merkmal identifiziert werden konnte, macht auf dem Hintergrund der Selbstbestimmungstheorie von Deci und Ryan Sinn. Danach ist das Bedürfnis nach sozialem Eingebundensein neben dem Bedürfnis nach Autonomie und nach Kompetenz eines der drei Basisbedürfnisse, die für das Entstehen einer selbstbestimmten Lernmotivation ausschlaggebend sind (Ryan \& Deci, 2000).

\section{Fazit}

Der Einfluss der sozialen und kulturellen Herkunft auf schulische Leistungen und die Bildungsbeteiligung konnte im Kontext von gegliederten Schulsystemen durch die vorliegende Analyse wesentlich präzisiert werden. Damit erhärtet sich der Befund, dass soziale Unterschiede gerade an den Übergängen als den Schlüsselstellen des Bildungswesens erhebliche Auswirkungen haben. Unser gegliedertes Bildungssystem behindert - gleiche Fach- und Sprachkompetenzen vorausgesetzt - nicht Schülerinnen und Schüler aus anderen Kulturen, sondern wirkt in erster Linie sozial diskriminierend. Bildungspolitisch bedeutet dies, dass zur Leistungsförderung und zur Reduktion der im internationalen Vergleich erheblichen Leistungsunterschiede eine gezielte Förderung sozial benachteiligter Schichten notwendig ist. Insbesondere sind die Einschulungsphase sowie die vielerorts noch vorzufindenden gegliederten Strukturmodelle auf der Sekundarstufe I und die damit verbundenen Übertrittsmechanismen zu überdenken.

Aus methodischer Sicht ist festzuhalten, dass - sowohl was die nationale Analyse als auch was den internationalen Vergleich betrifft - der Einbezug der kognitiven Grundfähigkeiten und des Schultyps für eine genauere Wirkungsbeschreibung der Herkunftsmerkmale und der Selektion notwendig ist. Die auf die 
soziale Herkunft zurückzuführenden Effekte sehen dann weniger dramatisch aus als undifferenzierte Roheffekte, sind aber weit besser validiert und immer noch statistisch gesichert und bedeutsam. Eine solche realistischere Einschätzung zeigt, wie die Schweiz zwischen sozialer Herkunft und Leseleistung zu einem gewiss nicht genetisch bedingt - engeren Zusammenhang kommt als fast alle anderen Länder.

\section{Anmerkungen}

1 In der Schweiz wurde zwar auch die Klassenzugehörigkeit erhoben; sie wurde aber nicht für eine Auswertung aufgearbeitet.

2 Eine detaillierte Beschreibung des Literacy-Konzepts findet sich in der PISA-Rahmenkonzeption für die Erfassung von Wissen und Fähigkeiten (OECD, 1999).

3 Ausser bei der Analyse der Schuleffekte wurde jeweils nur der erste der fünf plausiblen Werte (Mislevy, Johnson \& Muraki, 1992) in die Analyse einbezogen.

4 Der so gebildete Indikator beschreibt die komplexe Problematik der kulturellen Herkunft nur stark vereinfacht. Eine differenziertere Aufgliederung nach Herkunftsnationalität und Aufenthaltsdauer in der Schweiz brachte keine höhere Aussagekraft für die Datenanalyse, weshalb die einfache Gliederung beibehalten wurde.

5 Im Kanton Bern wird der gymnasiale Unterricht in der neunten Klasse nicht nur an Gymnasien, sondern auch dezentral an Sekundarschulen angeboten. Bei diesen Schulen kann nicht ausgeschlossen werden, dass eine Minderheit von Schülerinnen und Schülern, die zwar einer speziellen Sekundarklasse mit erhöhten Anforderungen angehören, jedoch den gymnasialen Unterricht nicht besuchen, den Gymnasien zugeordnet wurden.

6 Die Zahl fehlender Angaben ist so klein, dass mindestens 98\% der Fälle ausgewertet werden konnten, selbst wenn eine Person beim Fehlen einer einzelnen Angabe aus der ganzen Analyse ausgeschlossen wurde.

7 Modellbasierte, im Gegensatz zu Designbasierter Analyse (vgl. Lohr, 1999, 362ff.). Die Gewichtung der Daten entsprechend den Ziehungswahrscheinlichkeiten beeinflusst die Höhe der Korrelationen nur unwesentlich (max. $\Delta \mathrm{r}=.02$ ).

8 Neben den standardisierten Regressionskoeffizienten wird zu jeder Variablen der Anteil erklärter Varianz angegeben.

9 Aufgrund der in PISA verwendeten zweistufigen Stichprobe (zuerst Schulen, dann Schülerinnen und Schüler aus gewählten Schulen gezogen) wird der Stichprobenfehler mit Standardverfahren im Vergleich zu einfachen Zufallsstichproben unterschätzt. Gemäss der Analyse in Abschnitt Einfluss der einzelnen Schule auf die Leseleistung nimmt dieser Designeffekt (Kish, 1995) in der vorliegenden Stichprobe den Wert 2 an. Deshalb wurde die Stichprobengrösse für die Modellberechnungen in den Abschnitten Erklärungsmodelle zur Vorhersage vonschulischer Leistung und Chancenunterschiede beim Übertritt insGymnasium und die Sekundarschule halbiert. Auf diese Weise werden die statistischen Signifikanzen korrekt geschätzt. Angegeben wird jeweils die bereits halbierte Stichprobengrösse.

10 Für das Modell werden nur diejenigen Schülerinnen und Schüler berücksichtigt, die den PISA-Mathematiktest gelöst haben. Dies führt dazu, dass gegenüber den Modellen zur Erklärung der Leseleistung geringfügige Differenzen bei den Korrelationen der Einflussgrössen bestehen.

11 Die verbale Grundfähigkeit wird hier einbezogen, da es nicht (wie beim Modell M1 und den Modellen zur Erklärung der Leistung im vorherigen Abschnitt) darum geht, den Einfluss vorgegebener Grundfähigkeiten und sozialer Herkunft abzugrenzen, sondern den Einfluss der sozialen Herkunft auf den Übertritt auch bei möglichst vollständiger Kontrolle von Leistungen und kognitiven Fähigkeiten zu erfassen. 
12 Für alle auf Schulen bezogenen Analysen wurden sehr kleine Schulen mit weniger als 12 getesteten Schülerinnen und Schülern ausgeschlossen. Das trifft auf 12 Berner Schulen mit zusammen 46 Getesteten zu. In die Analyse wurden 61 Schulen und 1'972 Getestete einbezogen. Die Auswertungen wurden mit dem Programm HLM vorgenommen, wobei über die Ergebnisse der fünf plausiblen Werte gemittelt wurde.

$13 \mathrm{Da}$ es nicht darum geht, Herkunft und Grundfähigkeiten gegeneinander abzugrenzen, sondern ihre kombinierte Wirkung optimal zu kontrollieren, werden auch die verbalen Grundfähigkeiten einbezogen.

14 Da die Klassenebene nicht berücksichtigt wurde, kommen darin auch in bescheidenem, in der Restvarianz zwischen Schulen noch in vermehrtem Masse auf Klassen zurückzuführende Leistungsunterschiede zum Ausdruck.

15 Die Standardabweichung bezieht sich auf beiden Ebenen auf die Gesamtpopulation der Schülerinnen und Schüler.

16 Die Zahlenwerte entsprechen sich nicht ganz genau, da die Variable der sozialen Herkunft zwar sehr ähnlich, aber nicht identisch definiert wurde.

17 Zur Definition der Merkmale siehe OECD (2001) und Adams und Wu (2002).

\section{Literatur}

Adams, R. \& Wu, M. (Hrsg.). (2002). PISA 2000 technical report. Paris: OECD.

Artelt, C., Stanat, P., Schneider, W. et al. (2001). Lesekompetenz: Testkonzeption und Er-gebnisse. In J. Baumert, E. Klieme, M. Neubrand et al. (Hrsg.), PISA 2000. Basiskompetenzen von Schülerinnen und Schülern im internationalen Vergleich (S. 69-137). Opladen: Leske + Budrich.

Bain, D., Favre, B., Hexel, D., Lurin, J. \& Rastoldo, F. (2000). Hétérogénéité et différenciation au cycle d'orientation. Le débat genevois dans le contexte national et international: pratiques et recherches. Genève: Service de la recherche en éducation.

Baumert, J., Artelt, C., Klieme, E., Neubrand, M., Prenzel, M., Schiefele, U., Schneider, W., Tillmann, K.-J. und Weiss, M. (Hrsg.). (2003). PISA 2000. Ein differenzierter Blick auf die Länder der Bundesrepublik Deutschland. Zusammenfassung zentraler Befunde. Berlin: MaxPlanck-Institut für Bildungsforschung.

Baumert, J. \& Köller, O. (1998). Nationale und internationale Schulleistungsstudien. Was können sie leisten, wo sind ihre Grenzen? Pädagogik, 50, (6), 12-18.

Baumert, J., Köller, O. \& Schnabel, K.-U. (2000). Schulreformen als differentielle Entwicklungsmilieus - eine ungehörige Fragestellung? In M. Demmer (Hrsg.), Messung sozialer Motivation. Eine Kontroverse (S. 28-68). Frankfurt am Main: GEW.

Baumert, J. \& Schümer, G. (2001). Familiäre Lebensverhältnisse, Bildungsbeteiligung und Kompetenzerwerb. In J. Baumert, E. Klieme, M. Neubrand et al. (Hrsg.), PISA 2000. Basiskompetenzen von Schülerinnen und Schülern im internationalen Vergleich (S. 323-407). Opladen: Leske + Budrich.

Baumert, J. \& Schümer, G. (2002). Familiäre Lebensverhältnisse, Bildungsbeteiligung und Kompetenzerwerb im nationalen Vergleich. In J. Baumert, C. Artelt, E. Klieme et al. (Hrsg.), PISA 2000 - Die Länder der Bundesrepublik Deutschland im Vergleich (S. 159202). Opladen: Leske + Budrich.

BFS/EDK (Hrsg.). (2002). Für das Leben gerüstet? Die Grundkompetenzen der Jugendlichen Nationaler Bericht der Erhebung PISA 2000. Neuchâtel: Bundesamt für Statistik (BFS).

Binet, A. \& Simon, T. (1905). Application des méthodes nouvelles au diagnostic du niveau intellectuel chez des enfants normaux et anormaux d'hospice et d'école primaire. L'année Psychologique, 11, 245-336.

Boudon, R. (1974). Education, opportunity and social inequality. New York (NY): Wiley. 
Bourdieu, P. (1983). Ökonomisches Kapital, kulturelles Kapital, soziales Kapital. In R. Krekkel (Hrsg.), Soziale Ungleichheiten. Soziale Welt. (S. 183-198). Göttingen: Schwartz.

Breen, R. \& Goldthorpe, J. H. (1997). Explaining educational differentials: Towards a formal rational action theory. Rationality and Society, 9, (3), 275-305.

Cattell, R. B. (1971). Abilities: Their structure, growth, and action. Boston: Houghton Mifflin.

Coradi-Vellacott, M. \& Wolter, S. C. (2002). Soziale Herkunft und Chancengleichheit. In BFS/EDK (Hrsg.), Für das Leben gerüstet? Die Grundkompetenzen der Jugendlichen - Nationaler Bericht der Erhebung PISA 2000 (S. 90-112). Neuchâtel: Bundesamt für Statistik (BFS).

Dickens, W. T. \& Flynn J. R. (2001). Heritability Estimates Versus Large Environmental Effects: The IQ Paradox Resolved. Psychological Review, 108, 346-369.

Ditton, H. \& Krecker, L. (1995). Qualität von Schule und Unterricht. Empirische Befunde zu Fragestellungen und Aufgaben der Forschung. Zeitschrift für Pädagogik, 41, 507-529.

EDK (1997). Bildung und Erziehung der vier-bis achtjährigen Kinder in der Schweiz. Dossier 48A. Bern: Schweizerische Konferenz der kantonalen Erziehungsdirektoren (EDK).

Gamoran, A. (1992). Synthesis of Research: Is Ability Grouping Equitable? Educational Leadership, 50, (2), 11-13.

Gamoran, A. \& Berends, M. (1987). The Effects of Stratification in Secondary Schools: Synthesis of Survey and Ethnographic Research. Review of Educational Research, 57, 415-435.

Guthke, J. (1998). Intelligenz, Wissen und Lernfähigkeit. Zeitschrift für Pädagogische Psychologie, 12 (1), 5-9.

Heller, K., Gaedike, A.-K. \& Weinläder, H. (1985). Kognitiver Fähigkeits-Test KFT 4-13. Weinheim: Beltz.

Helmke, A., Hosenfeld, I., Schrader, F.-W. et al. (2002). Sozialer und sprachlicher Hintergrund. In A. Helmke \& R. S. Jäger (Hrsg.), Das Projekt MARKUS. Mathematik-Gesamterhebung Rheinland-Pfalz: Kompetenzen, Unterrichtsmerkmale, Schulkontext (S. 71-153). Landau: Verlag Empirische Pädagogik.

Helmke, A. \& Weinert, F. E. (1997). Bedingungsfaktoren schulischer Leistungen. In F. E. Weinert (Hrsg.), Enzyklopädie der Psychologie, Bd. 3: Psychologie des Unterrichts und der Schule (S. 71-176). Göttingen: Hogrefe.

Herrnstein, R. J. \& Murray, Ch. (1994). The Bell Curve. Intelligence and Class Structure in American Life. New York: Free Press.

Jöreskog, K. G. \& Sörbom, D. (1988). Lisrel 7. A guide to the program and applications (2nd ed.). Chicago: SPSS.

Kish, L. (1995). Survey Sampling. New York: Wiley.

Klauer, K. J. (1998). Anlage und Umwelt. In D. H. Rost (Hrsg.), Handwörterbuch Pädagogische Psychologie (S. 1-5). Weinheim: Beltz.

Lamprecht, M. \& Stamm, H. (1996). Soziale Ungleichheit im Bildungswesen. Bern: Bundesamt für Statistik.

Lohr, S. L. (1999). Sampling: Design and Analysis. Pacific Grove: Duxbury Press.

Lüdtke, O., Robitzsch, A. \& Köller, O. (2002). Statistische Artefakte bei Kontexteffekten in der pädagogisch-psychologischen Forschung. Zeitschrift für Pädagogische Psychologie, 16, 217-231.

Mislevy, R. J., Johnson, E. G. \& Muraki, E. (1992). Scaling Procedures in NAEP. Journal of Educational Statistics, 17, 131-154.

Moser, U. (2002). Kulturelle Vielfalt in der Schule: Herausforderung und Chance. In BFS/EDK (Hrsg.), Für das Leben gerüstet? Die Grundkompetenzen der Jugendlichen - Nationaler Bericht der Erhebung PISA 2000 (S. 113-135). Neuchâtel: Bundesamt für Statistik (BFS).

Moser, U., Keller, F. \& Tresch, S. (2002). Evaluation der 3. Primarschulklassen. Schlussbericht zuhanden der Bildungsdirektion des Kantons Zürich. Zürich: Kompetenzzentrum für Bildungsevaluation und Leistungsmessung an der Universität Zürich. 
Moser, U., Ramseier, E. \& Berweger, S. (2002). Die Grundbildung in den drei Kantonen. In BFS/EDK (Hrsg.), Bern, St. Gallen, Zürich: Für das Leben gerïstet? Die Grundkompetenzen der Jugendlichen - Kantonaler Bericht der Erhebung PISA 2000 (S. 17-34). Neuchâtel: Bundesamt für Statistik (BFS).

Moser, U. \& Rhyn, H. (1997). Evaluation der Sekundarstufe I im Kanton Zürich. Zweiter Bericht. Bedingungen des Lernerfolgs. Bern: Institut für Pädagogik der Universität.

Moser, U. \& Rhyn, H. (1999). Schulmodelle im Vergleich: Eine Evaluation der Leistungen in zwei Schulmodellen der Sekundarstufe I. Aarau: Sauerländer.

Moser, U. \& Rhyn, H. (2000). Lernerfolg in der Primarschule. Aarau: Sauerländer.

OECD (Hrsg.). (1999). Measuring student knowledge and skills. A new framework for assessment. Paris: OECD.

OECD (Hrsg.). (2001). Lernen für das Leben. Erste Ergebnisse von PISA 2000. Paris: OECD.

Plomin, R. (1988). The nature and nurture of cognitive abilities. In R. J. Sternberg (Hrsg.), Advances in the psychology of human intelligence (Bd. 4, S. 1-33). Hillsdale: Erlbaum.

Ramseier, E. (1997). Individuelle Determinanten der Mathematikleistung. In: U. Moser, E. Ramseier, C. Keller \& M. Huber (Hrsg.), Schule auf dem Prüfstand. Eine Evaluation der Sekundarstufe I auf der Grundlage der "Third International Mathematics and Science Study" (S. 113-136). Chur: Rüegger.

Rhyn H. (1995). Psychometrie und Bildung. Der Intelligenzquotient als Sozialindikator? Zeitschrift für Pädagogik, 41, 765-779.

Ryan, R. M. \& Deci, E. L. (2000). Self-determination theory and the facilitation of intrinsic motivation, social development, and wellbeing. American Psychologist, 55, 68-78.

Scheerens, J. \& Bosker, R. (1997). The Foundations of Educational Effectiveness. Oxford: Pergamon.

Stanat, P. (2002). Schulleistungen von Jugendlichen mit Migrationshintergrund: Die Rolle der Zusammensetzung der Schülerschaft. Jena: Vortrag an der 63. Tagung der Arbeitsgruppe für empirische pädagogische Forschung.

Weinert, F. E. (1997). Grenzen der Entwicklung des Humankapitals aus der Sicht der psychologischen Lerntheorie. In G. Clar, J. Doré \& H. Mohr (Hrsg.), Humankapital und Wissen: Grundlagen einer nachhaltigen Entwicklung (S. 137-156). Berlin: Springer.

Willms, J. D. (1992). Monitoring school performance. A guide for Educators. Washington: Falmer Press.

Zimmermann, P. \& Spangler, G. (2001). Jenseits des Klassenzimmers. Der Einfluss der Familie auf Intelligenz, Motivation, Emotion und Leistung im Kontext der Schule. Zeitschrift für Pädagogik, 47, 461-479.

Zutavern, M., Brühwiler, C. \& Biedermann, H. (2002). Die Leistungen der verschiedenen Schultypen auf der Sekundarstufe I. In BFS/EDK (Hrsg.), Bern, St. Gallen, Zürich: Für das Leben gerüstet? Die Grundkompetenzen der Jugendlichen - Kantonaler Bericht der Erhebung PISA 2000 (S. 63-76). Neuchâtel: BFS/EDK. 


\section{Perspectives de formation dans un système organisé en section : une analyse apporfondie des données PISA incluant les compétences cognitives des éléves.}

\section{Résumé}

Depuis la publication des résultats - plutôt décevants pour la Suisse - de l'enquête PISA, la question de l'équité de notre système éducatif est de nouveau à l'ordre du jour. Le présent article se propose d'analyser en détail la problématique de l'égalité des chances tout en tenant compte des capacités cognitives de base. Pour expliquer les différences de compétences entre les élèves, il convient non seulement d'examiner l'influence du milieu dont provient l'élève, mais aussi de se pencher sur le rôle joué par l'établissement scolaire. Notre analyse se fonde sur l'étude de 2054 élèves des cantons de Berne et de Saint-Gall ayant participé aux tests PISA et dont les capacités cognitives de base ont été recensées parallèlement. Les conclusions de l'étude montrent que le lien étroit entre les résultats scolaires et l'origine sociale existe même après contrôle des capacités cognitives de base. Elles soulignent par ailleurs le rôle joué par le type d'école ou la section fréquentée. Or l'appartenance à un type d'école dépend visiblement non seulement des résultats scolaires de l'élève, mais aussi du milieu social dont il provient. En outre, la composition socioculturelle de l'école influe sur les possibilités d'apprentissage de l'élève. Toutefois, ces effets de l'école sont beaucoup moins perceptibles lorsque l'analyse tient compte des compétences, du vécu individuel et du type d'école ou de la section que lorsqu'elle se limite à présenter les effets bruts, comme c'est le cas dans le rapport sur l'enquête PISA publié par l'OCDE en 2001 .

\section{Origine sociale, prestazioni scolastiche e opportunità formative in un sistema scolastico a filière verticali: analisi approfondita in considerazione delle capacità cognitive di base}

\section{Riassunto}

I risultati disincantati dell'inchiesta PISA hanno fatto sì che si incrementasse la discussione attorno all'equità del nostro sistema scolastico. In questo contributo si discutono in maniera dettagliata aspetti relativi alla parità delle possibilità e alle capacità cognitive di base e per spiegare le differenze dei risultati degli allievi si fa riferimento all'origine sociale e al fattore scuola. Nell'analisi sono entrati 2054 allievi provenienti dai Cantoni di Berna e di San Gallo a per i quali sono stata valutate, oltre a quanto richiesto da PISA, anche le capacità cognitive di base. I risultati mostrano che, pur considerando le capacità cognitive, le prestazioni a livello di singole discipline vengono condizionati tanto dall'origine so- 
ciale quanto dal tipo di scuola frequentata. Per converso si constata che il tipo di scuola in cui si trova l'allievo oltre che dalle prestazioni dipende in misura considerevole dall'origine sociale. Il livello di prestazione è pure influenzato dalla composizione culturale della scuola. Occorre però rilevare che, se sin tengono in conto le premesse individuali, questi effetti legati al tipo di scuola sono di portata minore rispetto a quelli indicati globalmente nel rapporto PISA (OECD, 2001).

\section{Origin, achievement and equal opportunity education in a multi type school system. A detailed examination of the PISA report taking into consideration basic cognitive competence.}

\footnotetext{
Summary

A consequence of last year's disappointing Swiss PISA results has been the revival of the discussion of equity in our education system. This article seeks to examine in detail aspects of equal opportunities whilst making allowance for basic cognitive competence. It is evident that pupils' academic achievement is influenced both by their socio-economic background and the features of their school. This analysis is based on data compiled from 2054 students in the Cantons of Berne and St. Gallen who, in addition to the PISA tests, were tested in their basic cognitive competence. The results demonstrate that academic achievement is strongly influenced by socio-economic background even when taking into consideration the influence of basic cognitive competencies. The most influential factor is the type of school or track a pupil attends. Attendance at a particular type of schools is not only linked to academic achievement but also, to a very large extent, to a pupil's socio-economic background. Furthermore, the opportunities for learning in an individual school are also influenced by the socio-cultural backgrounds of the pupils attending that school. However, when the analysis takes into consideration pupils' individual characteristics and the type of school, the differences between schools are much weaker than suggested in the international PISA report (OECD, 2001) which was based on gross-effects only.
} 